\title{
Generate, Repurpose, Validate: A Receptor-Mediated Atom-by-Atom Drug Generation for SARS-Cov-2 Spike Protein and Similarity-Mapped Drug Repurposing for COVID-19 with Rigorous Free Energy Validation Using Well-Tempered Metadynamics
}

Rituparno Chowdhury, Venkata Sai Sreyas Adury, Amal Vijay, Reman K. Singh, Arnab Mukherjee

Submitted date: 17/05/2020 - Posted date: 18/05/2020

Licence: CC BY-NC-ND 4.0

Citation information: Chowdhury, Rituparno; Adury, Venkata Sai Sreyas; Vijay, Amal; Singh, Reman K.; Mukherjee, Arnab (2020): Generate, Repurpose, Validate: A Receptor-Mediated Atom-by-Atom Drug Generation for SARS-Cov-2 Spike Protein and Similarity-Mapped Drug Repurposing for COVID-19 with Rigorous Free Energy Validation Using Well-Tempered Metadynamics. ChemRxiv. Preprint. https://doi.org/10.26434/chemrxiv.12318311.v1

Finding a cure for Covid-19 is of immediate and paramount importance. In this study, we propose new and repurpose drugs to prevent SARS-Cov-2 (Covid-19) viral attack on human cells. Our study comprises three steps: generation of new molecules, structural similarity mapping to existing approved and investigational drugs, and validation of their binding strengths to the viral spike proteins based on rigorous all-atom well-tempered metadynamics free energy calculations. We show that some of our new molecules and some of the existing drugs bind more strongly than human ACE2 protein to the viral spike protein. Therefore, these drug molecules may have the potential to be repurposed as a preventive therapy for Covid-19, subject to further experimental verifications.

File list (3)

MSCovid19May20.pdf (25.43 MiB)

view on ChemRxiv • download file

SICovid19May20.pdf (5.49 MiB)

view on ChemRxiv • download file

DNVLive_covid_SI_small.mp4 (46.73 MiB)

view on ChemRxiv • download file 


\title{
Generate, repurpose, validate: a receptor-mediated atom-by-atom drug generation for SARS-Cov-2 spike protein and similarity- mapped drug repurposing for COVID-19 with rigorous free energy validation using well-tempered metadynamics
}

\author{
Rituparno Chowdhury ${ }^{\dagger \neq}$, Venkata Sai Sreyas Adury ${ }^{\ddagger}$, Amal Vijay ${ }^{\ddagger}$, Reman K. Singh ${ }^{\ddagger}$, and Arnab \\ Mukherjee*
}

Indian Institute of Science Education and Research Pune, Maharashtra, India

KEYWORDS: Covid-19, de novo drug design, Spike protein, human ACE2, repurposing therapeutics, docking, molecular dynamics, free energy, well-tempered metadynamics

\begin{abstract}
Covid-19 has wreaked havoc over mankind. To find potential therapeutics, we have developed a method to generate de novo molecules that grow atom-by-atom optimizing their interaction energy with the receptor. With this method, we obtained several ligands that bind strongly to the receptor binding domain (RBD) of the viral spike protein and chose top 35 molecules $(\sim 0.3 \%$ percent of the number generated) based on the interaction energy. We also mapped the de novo molecules to approved and investigational drugs and chose 20 best candidates based on the docking score. We validated our molecules by calculating the computationally most accurate, albeit expensive, all-atom explicit-water free energy surfaces for all the 55 molecules along with hACE2 with RBD using well-tempered metadynamics simulations. We also show that our calculated free energy estimate of hACE2 and RBD is in close agreement with experiments. Additionally, we found several molecules ( 4 de novo and 5 drugs) that have comparable or stronger binding affinity to the RBD than that of the hACE2. This implies that these molecules and drugs could have the potential to block the RBD-hACE2 interaction and thereby prevent further infection in human cells. While the de novo molecules provide us with novel viral-entry inhibitors and will require extensive toxicological and pharmacological screenings, the drugs could be tested immediately for their efficacies against Covid-19. Among the drugs we obtained, the strong binding ones are Danoprevir, Glecaprevir, Paritaprevir (hepatitis C protease inhibitor), Saquinavir (HIV protease inhibitor) and Solithromycin (ketolide antibiotic used against community acquired pneumonia). This pool of drugs will allow strategic repurposing against Covid-19 for a particular prevailing condition.
\end{abstract}

\section{INTRODUCTION}

SARS-CoV-2, commonly known as the "Novel Coronavirus" or "COVID-19", is a positive strand RNA beta-coronavirus with large sequence similarities to the SARS-CoV and BatCov RATG13 RNA viruses. ${ }^{1}$ Despite being known from at least early last year ${ }^{2}$ to as far back as 2006 as found in a report by Tang et al. ${ }^{1}$, the virus, seemingly incurable and unstoppable, is in an exponential growth phase all across the world with numbers increasing every day. The approximate number of COVID-19 cases is $\sim 3.1$ million worldwide with $\sim 0.22$ million deaths by the end of April 2020. ${ }^{3}$. Without a cure or prevention, this virus could very well become much more than the highly morbid pandemic it currently is.

Therefore, it is imminent to find a cure for the virus. Coronavirus is encapsulated by a membrane full of trimeric spike proteins. This spike protein interacts with the peptidase domain (PD) of the human angiotensin-converting enzyme 2 (hACE2). ${ }^{4}$ Consequently, this spike protein has been alluded to as a potential target to design preventive and curative therapeutics. ${ }^{5}$ An alternate common target for viruses is the Main protease ( ${ }^{\mathrm{M}} \mathrm{Pro}$ ) and the Non-Structured Proteins (NSPs). For SARS-Cov-2 (Cov2), the high resolution crystal structure of CL3 protease, also known as ${ }^{\mathrm{M}}$ Pro, was resolved recently. ${ }^{6}$ However, simulation studies have indicated that the enzyme active site (the putative drug design target) of SARSCoV-2 ${ }^{\mathrm{M}}$ Pro is highly flexible making it less prone to be inhibited by common viral protease inhibitors. ${ }^{7}$ To find other potential targets, Zhou et al. $^{8}$ mapped protein-protein interaction network for proteins involved in Cov-2 and came up with many targets to be used for drug repurposing by docking the drugs from the DrugBank ${ }^{9}$.

The detailed structural elucidation of Cov2 and hACE2 (hACE2) interface by Shang et al. ${ }^{10}$ shows that the spike protein's RBD region remains to be the most important target for drug design for Cov2. The study identifies residues responsible for the interaction and tagged it as receptor binding motif (RBM). RBM is a part of a binding domain called RBD, which again is a part of the $\mathrm{S} 1$ region of the spike protein, whose entire structure was solved recently by Wrapp et al. ${ }^{11}$ This protein is composed of three subunits. Each of these subunits contains a receptor binding domain (RBD). In two of the subunits, the RBD is in the so-called "down" configuration and in one it is in the "up" configuration. However, the exact structure of the RBD complexed to hACE2 and the interactions involved in the site were 
redetermined very recently by Yan et al. by X-ray diffraction. $^{12}$

Although the spike protein is trimeric ${ }^{11}$ and exists in both open and closed forms ${ }^{13}$, it is the RBD of the monomer that is responsible for cellular recognition, its movement is also independent of the rest of the protein. ${ }^{14}$ Figure 1a shows the structure of the spike protein, where RBD is in the "up" configuration, in contact with hACE2. Figure $1 \mathrm{~b}$ shows the RBD separately to highlight the interaction hotspot (i.e., $\mathrm{RBM}^{10}$ ). The structure of RBM serves as the hotspot for a potential inhibitor of the interaction between spike protein and hACE2 peptidase domain.
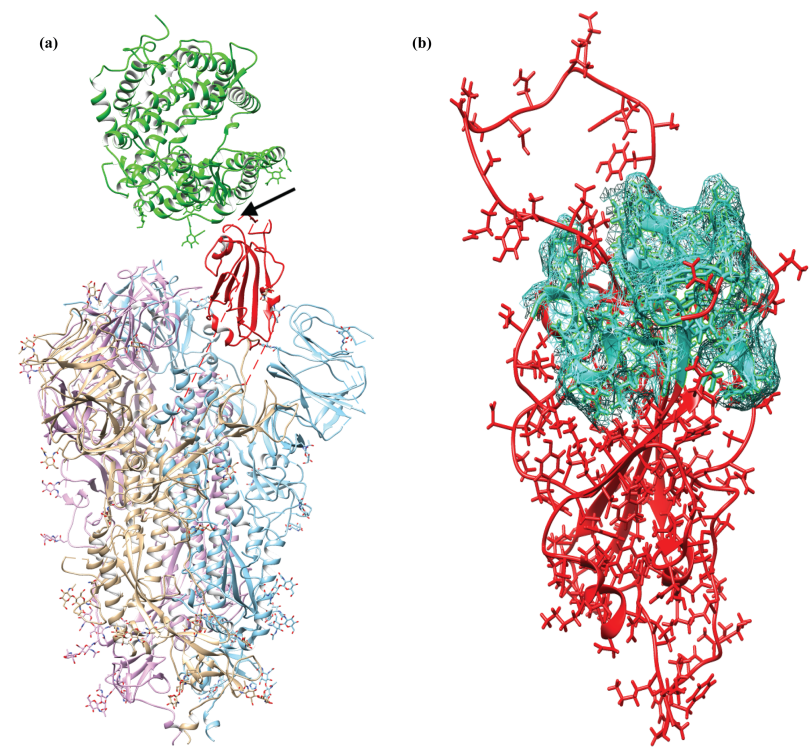

Figure 1. (a) The trimeric structure of the $\mathrm{S} 1$ region of the spike protein obtained from protein data bank with ID $6 \mathrm{VSB}^{11}$; RBD is shown in red and the hACE2 shown in green. The arrow indicates the target for a new inhibitor design. (b) The magnified version of the modelled RBD (in red) with the RBM (hotspot) shown as mesh surface in cyan.

The recent havoc created by the virus resulted in an upsurge of studies towards a remedy. Smith and co-workers performed ensemble docking of several drugs and identified some important ones that would bind to the RBD. ${ }^{15}$ This was followed by several docking studies targeting spike protein ${ }^{15-}$ ${ }^{16}$, Cov2 protease ${ }^{17}$, and hACE2 ${ }^{18}$, etc. All these studies resulted into a great number of possible drugs which could be repurposed for the treatment of Covid-19, pending a rigorous theoretical or an experimental tests.

Although docking is a quick method to sieve plausible candidate for binding, it often fails to ascertain the correct binding constant due to lack of water, ions, and entropic effects due to fluctuations of proteins, ligands and water molecules. ${ }^{19}$ Therefore, docking gives a wide range of molecules to try and often it becomes difficult to choose the best from them. Despite these pitfalls, docking gives a quick method for finding repurpose drugs. ${ }^{20}$ The most reliable and accurate approach to estimate binding constant, computationally, is to calculate it from an all-atom, explicit water simulation. ${ }^{21}$ To the best of our knowledge, no attempt has been made to find out the binding constants of any of the ligands through such rigorous methods for any of the target proteins for Covid-19.
The competitive inhibition by the drug will work if the RBD binds to the drug stronger than it does to hACE2. Therefore, estimating the binding constant accurately is essential. Using surface plasmon resonance experiments, Shang et al. reported a value of $44.2 \mathrm{nM}(-10.2 \mathrm{kcal} / \mathrm{mol}$ at $300 \mathrm{~K})$ binding affinity for the his-tagged RBD by covalently immobilizing the protein to the sample substrate. ${ }^{10}$ However, noncovalent association methods, which underestimates the $\mathrm{K}_{\mathrm{d}}$, provide a lower value of $2.9 \mathrm{nM}^{13}$ and $1.2 \mathrm{nM}^{13}$. The $\mathrm{K}_{\mathrm{d}}$ measured by Wrapp et al. using biolayer inferometry is $34.6 \mathrm{nM}^{11}$ The above values when converted to free energy at $300 \mathrm{~K}$ temperature will yield a range between -10.3 and -12.3 $\mathrm{kcal} / \mathrm{mol}$. We will show later that our computational estimate of the above is very close to the above experimental result.

Therefore, our primary aim for this study is to find a molecule that can bind to the hotspot of RBD with binding free energy lower than $-10.3 \mathrm{kcal} / \mathrm{mol}$. For that, we have developed a de novo molecule generation program, called 'DeNovo', that creates molecules atom-by-atom in the protein's hotspot (a defined structural region of a biomolecule) to optimize the interaction energy between the two. The idea of such a de novo generation stems from the fact that the chemical space is infinite $^{22}$ and there are molecules in our chemical space that would strongly bind to a given receptor, if only we can find them. Although the program is quite general and can grow molecules for any hotspot, we apply it for the first time here to grow inhibitors for the RBD by targeting the RBM region (Fig. 1b). Our approach will be particularly helpful for creating inhibitors where existing drugs have already started facing resistance and a completely new design for a drug is essential. $^{23}$ The current problem of Covid-19, however, demands a different solution, where we need to provide molecules worthy of immediate clinical trials.

Here we have generated several de novo molecules and found analogous drugs from the DrugBank ${ }^{9}$ that could be repurposed for Covid-19 using a similarity-based mapping. We have validated our approach by performing the computationally rigorous all-atom, explicit water well-tempered metadynamics $^{24}$ free energy calculations and show that 9 molecules ( 4 de novo and 5 drugs) have free energy of binding to the RBD lower than -10.3 (lower than the cut-off set above), implying their highly promising potential to inhibit the viral attack on human protein. As the benchmark, we calculated the free energy of binding between RBD-hACE2 in good agreement with experiments.

Therefore, our study provides a new, viable, and successful approach for finding novel and repurposed drugs for Covid19 , although it has the potential to be used for any other receptor-mediated drug design as well.

\section{RESULT AND DISCUSSION}

A. Generation of specific binders for a receptor. Our DeNovo program (see Method), schematically shown in Fig. S1 of the supporting information (SI), generated 13516 molecules having 10 to 50 heavy atoms to cover a broad spectrum of molecular scaffolds. The distribution of interaction energy between the molecule and the RBD is shown in Fig. 2. Once fitted to a standard Gaussian function, the mean comes at $-27 \mathrm{kcal} / \mathrm{mol}$ and standard deviation at 11.5 $\mathrm{kcal} / \mathrm{mol}$. We have chosen $\sim 0.3 \%$ of the molecules by selecting those that have interaction energy less than a cut-off 
value of $-61.5 \mathrm{kcal} / \mathrm{mol}$ (Fig. 2) set at three standard deviations lower than the mean energy.

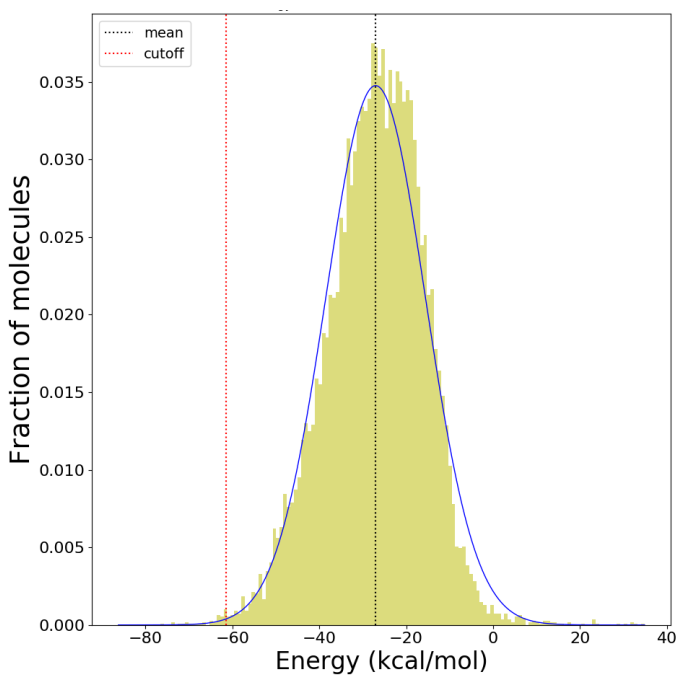

Figure 2. Distribution of interaction energies with RBD for 13516 DeNovo generated molecules. The mean and standard deviations of the fitted Gaussian distribution is $-27.0 \mathrm{kcal} / \mathrm{mol}$ and 11.5 $\mathrm{kcal} / \mathrm{mol}$, respectively. The cut-off (redline) is set at three standard deviations lower than the mean, i.e., at $-61.5 \mathrm{kcal} / \mathrm{mol}$.

Thus we gathered 35 molecules, plotted in Fig. S2 of SI. The names of the molecules are chosen based on an internal criteria of numbering. At this point, we have a set of good molecules to work with. However, our molecule generation had a few caveats: (1) our hotspot was rigid, (2) there were no water molecules (partially accounted for by using dielectric constant), (3) and the entropy contributions could not be taken into account. Therefore, to calculate the exact binding free energy, to the extent that a classical force field can provide, we have subjected the aforementioned drugs to a state-of-the art rigorous well-tempered ${ }^{24}$ version of metadynamics ${ }^{25}$ simulation for all these molecules as described later.

B. Route to repurposing. As we will see later, the DeNovo generated molecules are indeed strong binders and some of which could be candidate drugs. However, synthesis and toxicology study for these molecules may take much longer. Therefore, under the current state of urgency, we need readily available drugs that can be repurposed for Covid-19. Relying on the capability of our de novo molecules and building on the concept that similar molecules have similar chemistry ${ }^{26}$, we looked for similar molecules in the DrugBank ${ }^{9}$ using Tanimoto (or Jaccard) ${ }^{27}$ similarity search which uses bitwise fragment comparison to accurately match structures based on chemical fragments and motifs present in the input structures ${ }^{2}$. This similarity algorithm generally preserves relative positions of functional groups. We have chosen drugs for each of our 35 molecules with the Tanimoto coefficient $\geq 0.4$, following the recommendation of Baldi et al. who showed that a Tanimoto score of 0.4 is significant for a database of over 10,000 molecules. $^{28}$ This similarity search led to several drug molecules for each of the 35 de novo molecules, listed in Table S1 with their similarity score in brackets. After removing the irrelevant drugs (shown in red), such as the ones in the category of narcotics, anaesthetics, illicit substances, etc. (see the full list in SI), we ended up with 123 unique drugs. Given the difficulty and time required to perform free energy calculations for all these molecules, we docked these all the drugs to the same hotspot (Fig. S3 of SI), using docking score purely as a sieving criterion, and selected molecules with a docking score $<-8.0 \mathrm{kcal} / \mathrm{mol}$. This narrowed down our list of potentially repurposable drugs to 20 molecules. Figure S4 of SI shows the chemical structures of the chosen drug molecules and their docking scores are given in Table S2. As docking score varies even with the same parameters based on the representation of the drug (see Table S3 of SI and related discussion), we carried out the free energy simulations of these drug molecules with well-tempered ${ }^{24}$ metadynamics method, as well.

To capture the relationships between the de novo and drug molecules, we plot the similarity matrix of all the 55 molecules in Fig. 3. This matrix obtained by measuring similarity-based clustering of each pair of molecules. This matrix provides valuable information on the structural diversity of molecules that have led to favourable properties. We find that there are two separate clusters, one dominated by repurposed drugs and the other dominated by de novo molecules. 


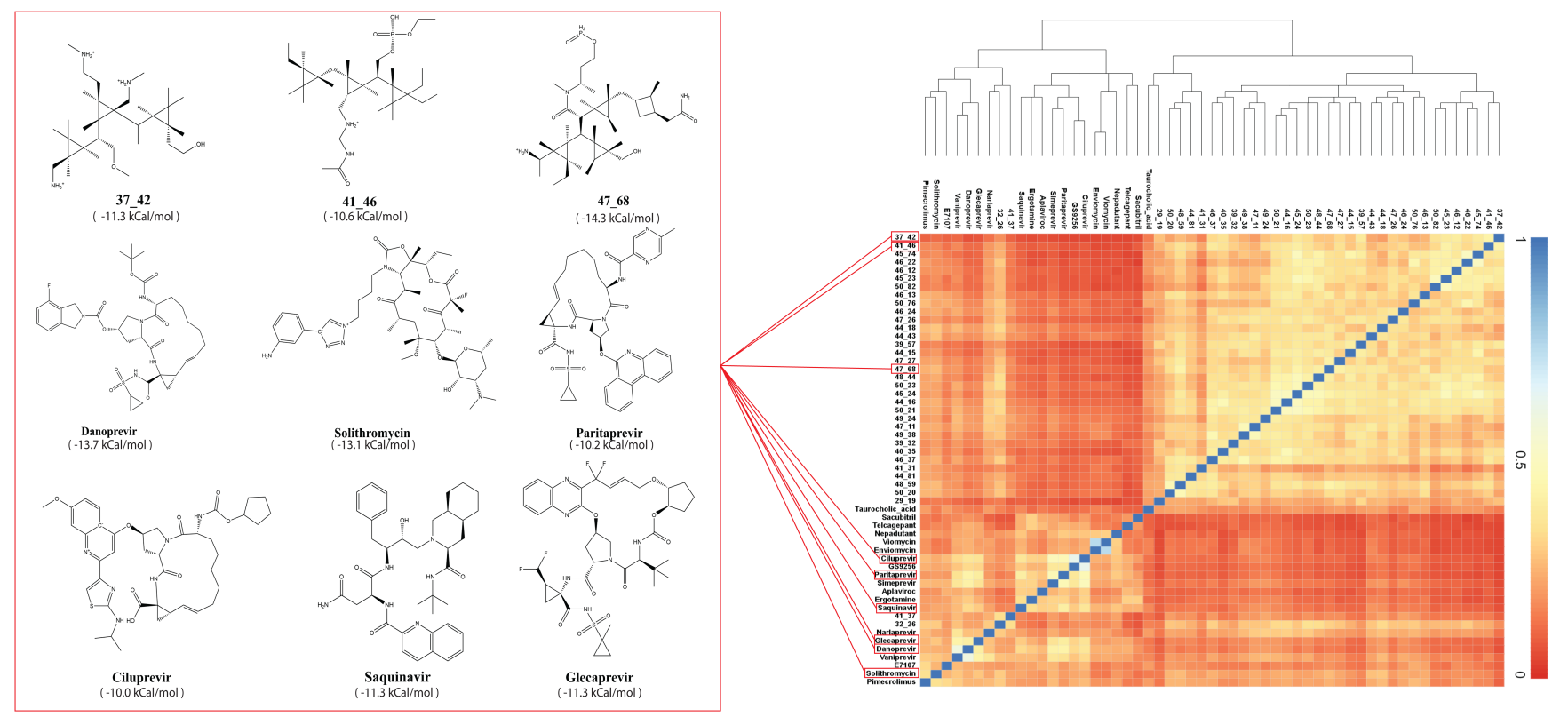

Figure 3. Heat map of the Tanimoto similarity amongst the chosen list of de novo and drug molecules. The hierarchical diagram also depicts the similarity between different molecules. Two clusters of de novo molecules and drugs are clearly visible. However, there are cross similarity also present.

We note that the molecular similarity reflects on the similarity in binding free energy as well. The best-binding de novo molecules (as scored based on DeNovo's interaction energy) are in themselves quite similar, as are the best-binding repurposed drugs in themselves. This is particularly noticeable once the molecules have been mapped to their free energies, which is done for those molecules with $<-10.0$ $\mathrm{kcal} / \mathrm{mol}$ in the left panel of Fig. 4. Furthermore, even though it may seem at the first glance that the cross-similarity is less, there are marked, and sporadically distributed regions of cross-similarity observed between the de novo molecules and repurposed drugs, which can be attributed to appreciable similarities in their maximal common substructures.

The left panel of Fig. 3 shows the structures of some of the best molecules along with their free energy (see below). It is truly reassuring that we obtained several molecules, both from our de novo generation and repurposing strategy, that are comparable or stronger in binding to RBD than hACE2.

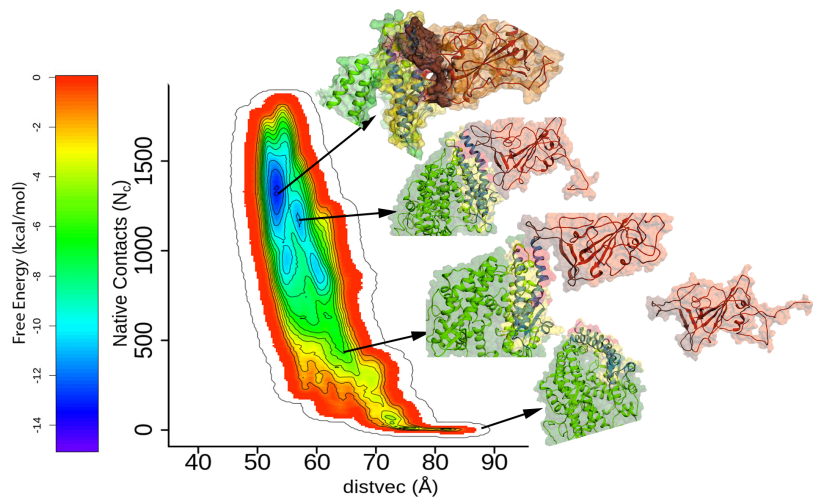

Figure 4. Free energy surface of RBD binding to hACE2 against two collective variables, native contact $\left(N_{C}\right)$ and distvec $(\AA)$. The structures along the path of dissociation are shown along the free energy bar diagram.
C. Free Energy Surfaces of Protein-Protein and ProteinDrug Complexes: An induced fit Mechanism. Free energy calculation with all-atom explicit water system is by far the most accurate, albeit expensive, estimate of binding among various computational methods. ${ }^{21}$ Metadynamics is an extremely popular state-of-the-art method to calculate free energy surface for complex systems ${ }^{29}$ and it has been shown to reproduce experimental observations closely ${ }^{30-31}$. Recently, attempts to estimate free energy of binding was done for the protease, albeit with an approximate method called $\mathrm{MM} / \mathrm{PBSA}^{32}$, which takes water as continuum, thus leading to an inaccurate estimation of entropy, and furthermore due to its lack of entropic effects it is also not ideally suited for providing mechanistic insights. All-atom with explicit water free energy calculation not only estimates the free energy more accurately, it also captures the mechanism of the binding. To the best of our knowledge, we are the first to perform the free energy calculations for the spike protein's interaction with hACE2 and other ligands. Here we have covered a huge list of 55 ligand molecules ( 35 are de novo and 20 are known drugs).

Before calculating the free energy for all the 55 drugs molecules, we validated our approach by calculating the free energy surface for RBD and hACE2 for comparison. Starting with the available crystal structure of the spike protein and hACE2 (PDB id $6 \mathrm{VSB}^{11}$ ), we first selected only the RBD region, modelled the loop region (see method), solvated the system with water and physiological concentrations of ions and performed multiple, long ( $230 \mathrm{~ns})$ well-tempered metadynamics simulations against multiple collective variables such as native contact (Fig. S5 of SI) and distvec (Fig. S6 SI) to study the binding free energy surface of these two proteins. While native contact helps to untangle the interactions between two proteins, distvec is a vectorial distance that helps move the proteins apart. These coordinates were used successfully in our previous studies of drug 
intercalation $^{33-34}$ and protein-protein interactions ${ }^{35}$ in our group.

Figure 4 shows the free energy surface which verifies the crystal structure configuration as the global free energy minimum. A few snapshots of the configuration of both proteins are shown along the unbinding pathway which depicts that the major reason for stability is due to direct protein-protein interaction. As soon as the proteins detach themselves, the free energy increases pushing the proteins apart, reflected in the increase in distvec. From this free energy surface, we get the binding free energy to be -13.3 $\mathrm{kcal} / \mathrm{mol}$ which is in close agreement with the experimental results. A second metadynamics simulation of the same system yielded free energy estimate of $-12.7 \mathrm{kcal} / \mathrm{mol}$ making the average $-13.0 \mathrm{kcal} / \mathrm{mol}$.
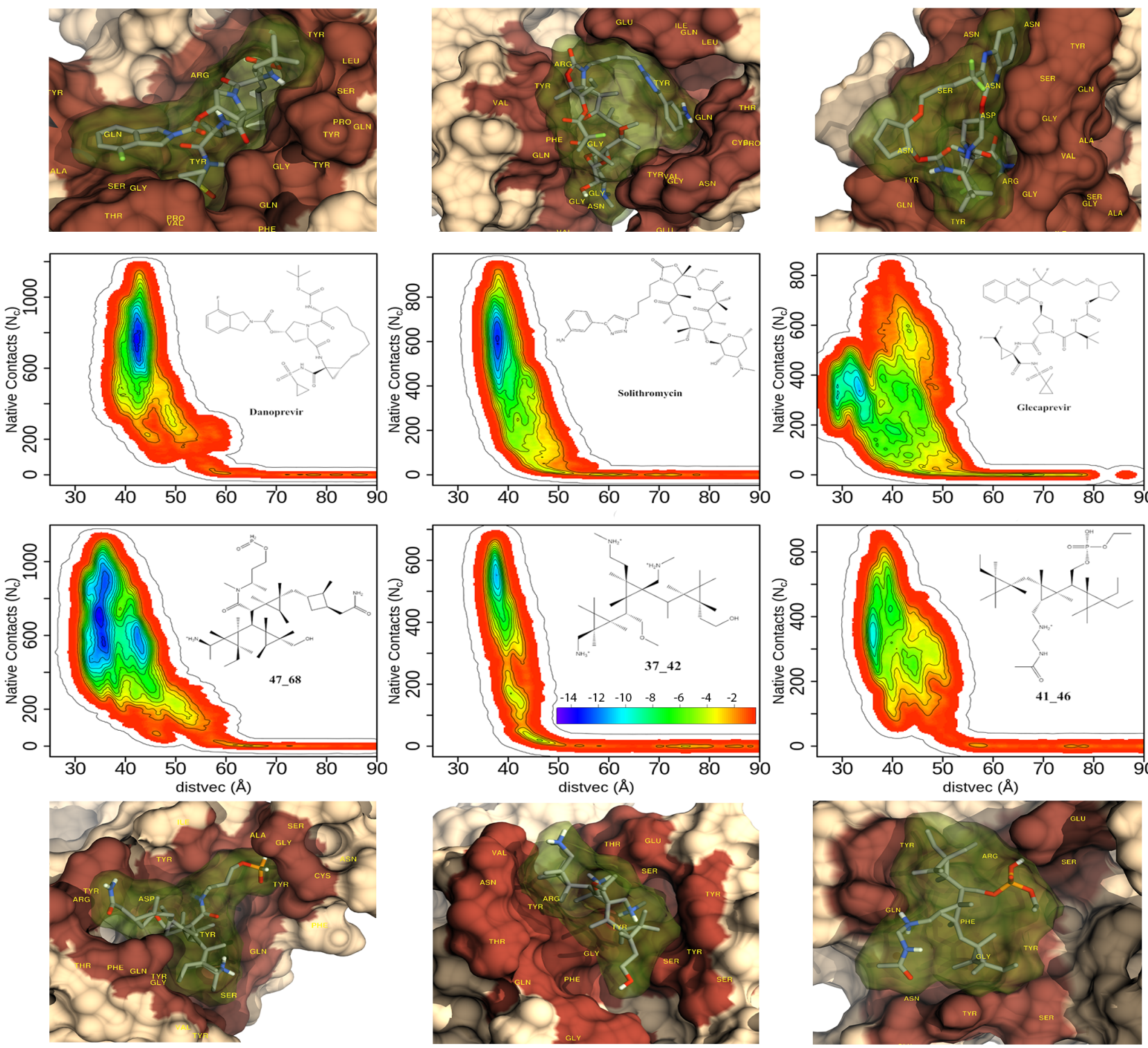

Figure 5. Free energy surfaces (FES) of three best de novo molecules and three best drug molecules in terms of free energy of binding. The chemical structure and the name of each molecule are shown in the inset. The free energy bar used to plot FES is shown in the inset of 37 42. The structures of the free energy minimum of each molecule, made using chimera ${ }^{36}$, are shown above/below the corresponding FES's. The protein is drawn in surface representation while the drug is drawn with both stick and surface (with $80 \%$ transparency). Note the deep cavity formed by each of these molecules in the protein.

Using the same set of collective variables and starting with either the docked state for drugs or the DeNovo generated configurations for the novel molecules, we performed metadynamics for all the 55 molecules and calculated their free energy surfaces.

Figure S7 to S10 in SI show the two-dimensional free energy surfaces of binding of all the molecules. In Fig. 5, we show the FES of three top de novo molecules and 3 top drugs. A representative structure of the most stable configuration obtained from the metadynamics simulation is shown either above or below the FES of the respective molecule. Figure 5 shows that the molecules are nicely packed inside the hotspot region.

Since each molecule behaves differently, it is not possible to find a unifying trend in their binding mechanism. However, most of the strong binding molecules have a narrow free 
energy profile along the native contact. Once the contacts (the short distance between the ligand and the protein) are broken, the separation between the ligand and protein quickly increases.

We summarize the results from our metadynamics simulations of all the systems (55 molecules and hACE2) in Fig. 6. The details of the system size and the runtime are given in Table S4 of SI. The free energies of the molecules are shown as vertical bar, where the RBD-hACE2 free energy values (experimental and computational) are shown as horizonal bars. We provided error estimates for the free energy for the hACE2 and some of the strong binding drug molecules by performing multiple free energy calculations. Different experimental techniques provide different estimates for the strength of hACE2 binding to the spike protein, as represented by the grey bar in Fig. 3. However, the lower limit of the interaction is $-12.3 \mathrm{kcal} / \mathrm{mol}$ which is close to our calculated value of $-13.0 \mathrm{kcal} / \mathrm{mol}$. The vertical bars show the binding free energy of 35 de novo molecules and 20 drug molecules. We can see that at least 9 molecules touch or cross the experimental bar indicating that RBD would bind to these molecules comparably or stronger than hACE2. Three molecules (47 68, danoprevir and solithromycin) supersede even the lowest estimate of binding strength of hACE2 with RBD.

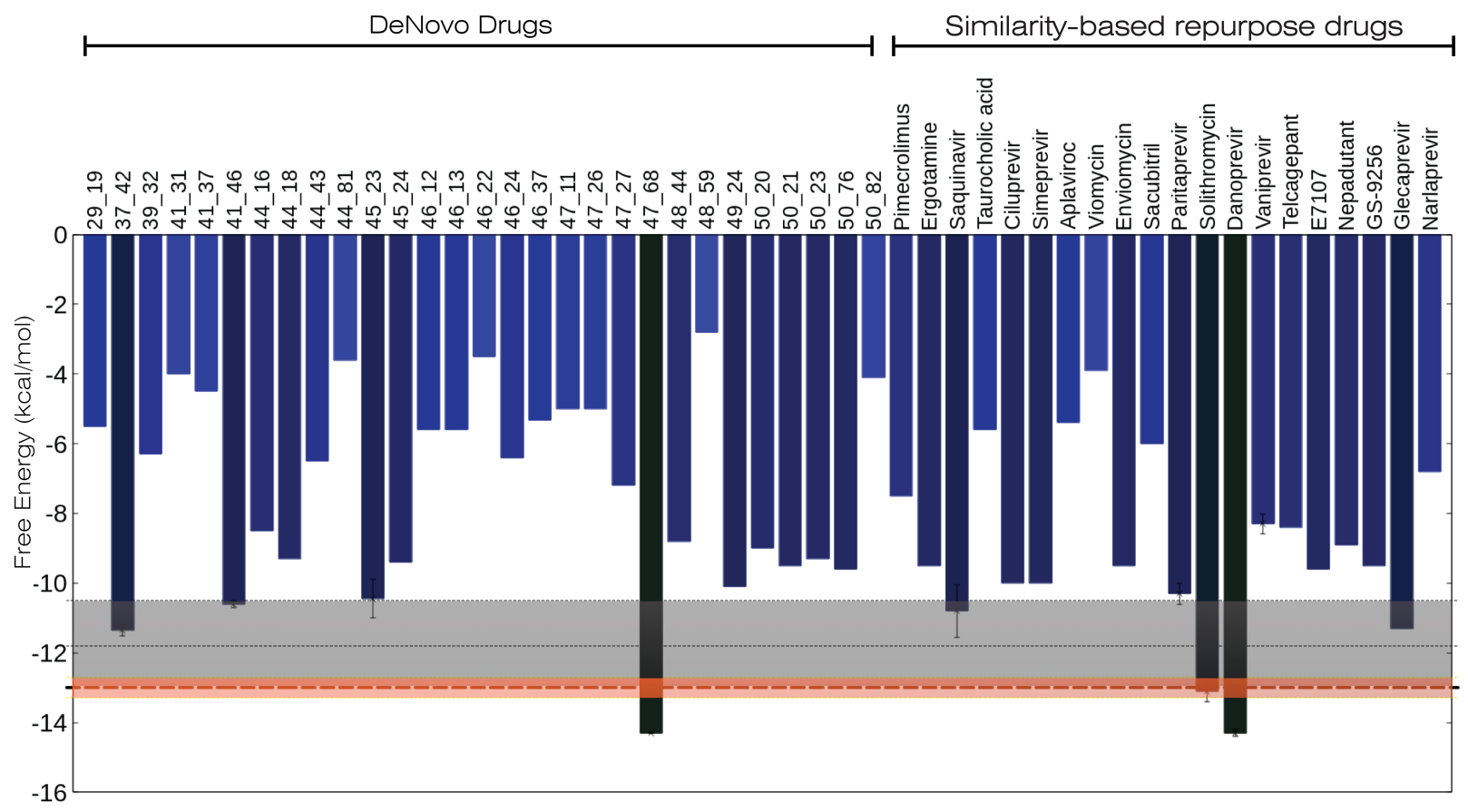

Figure 6. Free energy of binding for all the 35 de novo molecules and 20 drugs to RBD calculated using all-atom, explicit water, welltempered metadynamics simulations. The horizontal grey bar indicates the experimental range of free energy of binding of RBD and hACE2. The orange bar is the free energy estimate from multiple metadynamics simulations of RBD and hACE2. Error bars, obtained using multiple metadynamics simulations, are shown for some of the strong binding molecules. Note that, with RBD, may molecules bind comparably or stronger than hACE2.

Figure 5 shows that the strong binding molecules create a cavity within the protein. To understand this better, we investigated the flexible loop (residues 445 to 468) around the hotspot region. We have introduced an angle $\theta$ (see Fig. S1 1 of SI) that categorizes the configuration of the loop near the hotspot into three distinct conformations - close, open, and semi-open. We calculated the distribution of $\theta$ from the metadynamics simulations for all the systems including the free protein and hACE2 bound states. We plot the distribution for the most stable ligand, free protein and hACE2 bound protein in Fig. 7. This distribution shows that peaks at high value $\left(155^{\circ}\right)$ in the hACE2 bound RBD configuration, attributed to the open configuration. The distribution of is around $80^{\circ}$ in the free state, characterized here as the semi open state while the distribution of for the most stable ligand bound $\mathrm{RBD}$ is around $60^{\circ}$. The loop configurations for the other ligand bound states lie between the two extremes of $55^{\circ}<\theta<155^{\circ}$ (between hACE2 and danoprevir bound states). The $\theta$ for all the other ligand-bound states are shown in Fig. S12 of SI. 


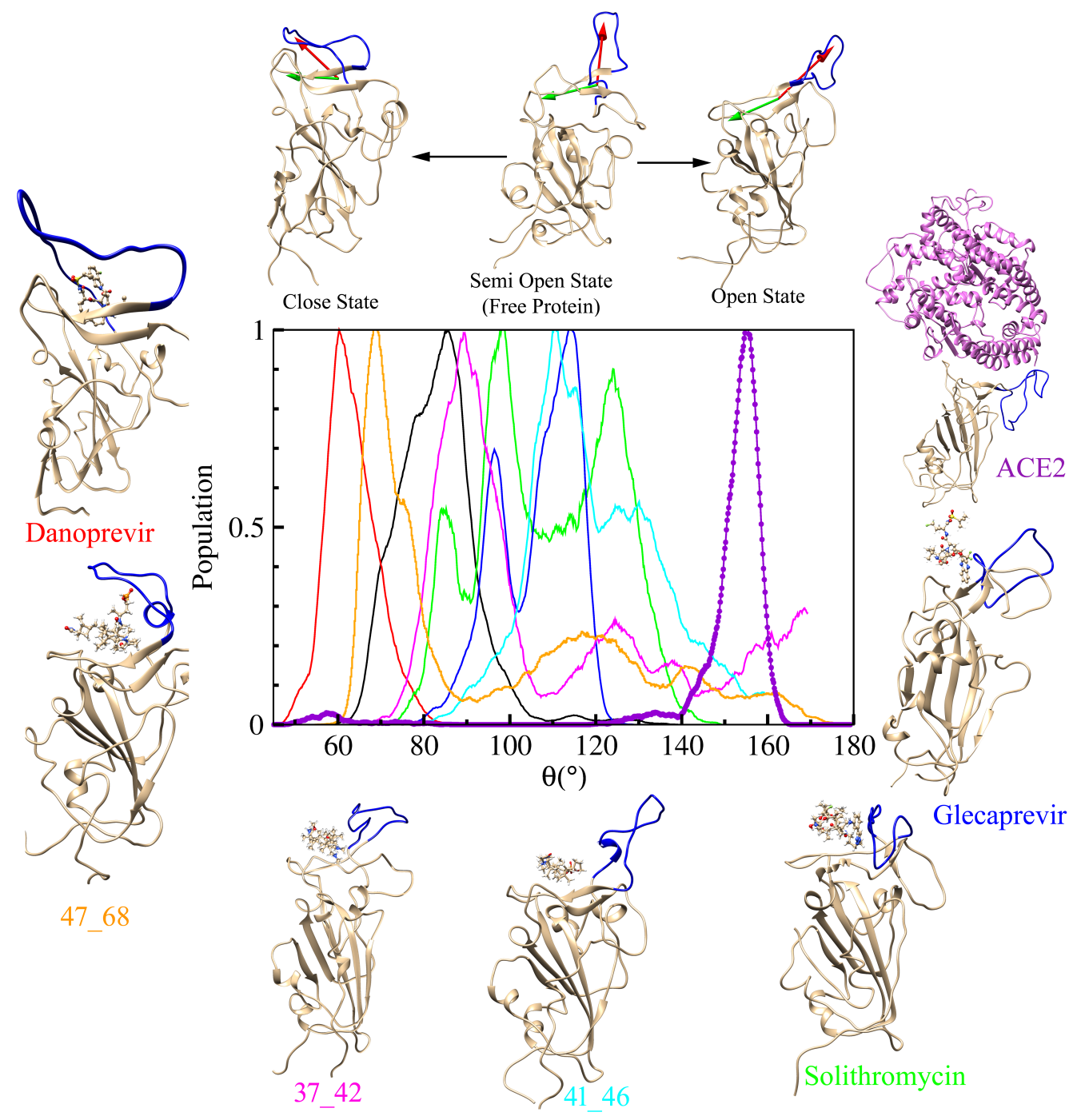

Figure 7. Distribution of loop configuration in terms of the angle between the red and green vectors (see Fig. S11 of SI) from the metadynamics simulation. The top panel shows three categories of loop configurations: open, semi open and close states. Representative images of the most stable ligand-bound states are shown where the structures are arranged from open to close configurations in clockwise fashion. Note that, while free protein belongs to the semi open configuration, the hACE2-bound and danoprevir (and 47_68)-bound RBD lie in the two extremes -- open and close states, respectively.

The above distribution indicates that binding of RBD to hACE2 and the ligands discussed here generate very different protein configurations. Therefore, upon binding of these ligands, the RBD configuration will be different and it will not be able to interact with the hACE2 effectively, achieving the competitive inhibition.

\section{CONCLUSION}

In this study, we have targeted a particular receptor, namely the receptor binding domain (RBD) of the spike protein, and designed several molecules through an atom-by-atom de novo generation protocol that tries to optimize the interaction of those molecules with the receptors. We selected only top $0.3 \%$ molecules (35 in number) and carried out free energy calculations using all-atom models of protein, ligand, water and ions using state-of-the-art well-tempered metadynamics method. The success of our de novo method is validated when 10 molecules have binding free energy to $\mathrm{RBD}<-9 \mathrm{kcal} / \mathrm{mol}$ ). We then mapped each of the molecules to approved and

investigational drugs in the DrugBank ${ }^{9}$ based on structural similarity and picked up the top 20 based on docking score. The all-atom free energy calculations were also performed with these drug molecules to the RBD. Out of the 20 molecules chosen, 11 of them bind to RBD with binding free energy below $-9 \mathrm{kcal} / \mathrm{mol}$. To validate our free energy method, we also calculated free energy of hACE2 with RBD and found it $(-13.0 \mathrm{kcal} / \mathrm{mol})$ in close agreement with experiment $(-12.3 \mathrm{kcal} / \mathrm{mol})$. Overall, we found at least four de novo molecules and five drugs molecules that bind to RBD comparable or stronger than the hACE2. This implies that these molecules could in principle inhibit the interaction of the RBD with hACE2 and prevent the viral attack on the human cell.

These repurposed drugs are molecules which seem to have by themselves a rich history as drugs for several threatening and even currently looming diseases. Ciluprevir, Glecaprevir and Paritaprevir are all hepatitis virus NS3/4A inhibitors, designed based on the transition state intermediates in the 
enzymatic active site with promising remedial properties as a small molecule inhibitor of the hepatitis infection. The possible binding mechanism of these drugs are generally mediated by $\alpha$-keto or sulfonate-based electrophilic warheads $^{37-38}$, disrupting key interactions in the nucleophilic NS3/4A enzymatic cavity. Apart from Glecaprevir, both Ciluprevir and Paritaprevir are administered alongside CYP3 inhibitors to improve their cell permeability and lower their IC50 dosage. On similar lines, Danoprevir and Saquinavir were also transition state analogue-based inhibitors for the NS3 protease, and are made to competitively inhibit the gagpol substrate. ${ }^{39-40}$ Solithromycin is an anti-microbial ${ }^{41}$, antiinflamatory ${ }^{42}$ drug that has been used in several occasions for decades $^{43}$; its role as an antiviral is known for respiratory tract infections ${ }^{44}$, apart from being a potent inhibitor to a host of other diseases ${ }^{43}$. This multi-functionality that we report for the aforementioned drugs is something be expected due to the immense number of secondary and even tertiary roles a drug molecule has once it enters the human body. As a study by Baker et al. eloquently shows, around 35,000 of all known drugs and drug-like substances worldwide (belonging to different chemical classes) have been used in more than one disease, while roughly 189 such molecules have been used as drugs for over 300 diseases. $^{45}$

Therefore, in this study, we not only established a protocol of finding new molecules for any given receptor, we also provide a definitive way to find and repurpose drugs in-silico. We have also validated our finding to the widely accepted and rigorous free energy protocol currently present in the literature. We hope that experiments carried out with the molecules presented here will further validate our results and it will be useful for our ongoing endeavour to fight against COVID-19 pandemic.

\section{METHODS}

A. DeNovo: The atom-by-atom synthesis of a strong binder. Here we describe our algorithm of de novo atom-by-atom construction of molecules in the hotspot of a receptor. The algorithm is based on the fact that any molecule can be represented as a graph where nodes are atoms and the bonds are edges. The first evidence of graph representation of the molecules dates back to 1867 by Kekule ${ }^{46}$. Recently, graph based algorithm was used to create 166 billion molecules in vacuum from only 17 atoms ${ }^{47}$ of $\mathrm{C}, \mathrm{N}, \mathrm{O}, \mathrm{S}$, and halogens, which reinforces the fact that the chemical space is infinite and therefore it is possible to get possibly multiple strong binders for each hotspot.

$\mathrm{We}$, however, grow the molecules in the protein hotspot from the similar set of atoms (C, N, O, S, etc.). The classical nonbonding interaction energy (both van der Waals and electrostatic) between the drug and the hotspot is calculated using CHARMM $27^{48}$ force field at each stage and the generation proceeds following an algorithm similar to that of the configuration bias Monte Carlo (CBMC $)^{49}$. Prior to molecular generation, the incomplete residues were completed using xleap of AMBERTools ${ }^{50}$ and the missing residues of the protein were modelled by Modeller $9.21^{51}$. We targeted a prefixed atom number for the molecule and tried to improve the interaction of the molecule using CBMC criteria. This way, we obtained molecules having between 10 to 50 heavy atoms. Unlike in CBMC, our molecule is made of many different atoms. We used geometric criteria (equilibrium distance and angles) for the formation of rings. Finally, even when a molecule reaches the desired number of atoms, CBMC protocol was used to create different molecules of similar size. The program is fully CPU-parallelised and runs efficiently over several cores (here we used 48 cores for our molecule generation). The schematic growth profile is shown in S1 of the supporting information (SI) and a video of a selected section of the generation suitable for conveying all the key points of the algorithm is provided as $\mathrm{SV} 1$ of SI.

B. Similarity Measurement. We have chosen all de novo generated molecules that interact with the protein with energy lower than our cut-off $-61.5 \mathrm{kcal} / \mathrm{mol}$ (see Fig. 2). Then we performed a similarity search using the DrugBank ${ }^{9}$ search engine employing Tanimoto algorithm ${ }^{52}$. All approved and investigational drug molecules with similarity above 0.4 , a cutoff based on the studies of Baldi et al. ${ }^{28}$, were considered. Table S1 of SI list all the drug molecules for each of the 35 de novo molecule that match the criterion.

C. Docking of the matched drugs. Based on the similarity measurement, we came up with a list of 123 unique drug molecules from our 35 de novo molecules. We then docked these drug molecules using AutoDock Vina version 1.1.253. in the hotspot. A box centered around SER443 is created with dimensions $36.7 \AA$ x $26.1 \AA$ x $40.9 \AA$ and default vina parameters. The docking setup is shown pictorially in Fig. S3. Input files of the drugs are created by converting PDB files to PDBQT using OpenBabel 3.0.054. The docking scores for all these molecules are shown in bracket in Table S1. The drug molecules with docking score less than $-8.0 \mathrm{kcal} / \mathrm{mol}$ were considered for free energy calculations.

D. Force Field Generation. All drug molecules were optimized using HF (Hartree Fock) theory with 6-31G* basis set using Gaussian 09 software ${ }^{55}$. Thereafter, antechamber ${ }^{56}$ module of AMBER $18^{50}$ is used for the RESP charge calculation of drug atoms. The forcefield was generated using GAFF forcefield ${ }^{57}$. The topology and coordinates were then converted into the GROMACS format by using a python script acpype.py (available at https://github.com/t-/acpype).

E. System Setup for Simulation. The starting structure of the SARS-CoV-2 spike glycoprotein (PDB ID: 6VSB) was obtained from the Protein Data Bank. Modeller 9.21 ${ }^{51}$ was used for modeling the missing residues, which predicted five number of three-dimensional structural forms using chain A of protein as the template. The best possible structure was predicted considering the DOPE (Discrete Optimized Protein Energy) score ${ }^{58}$. All of the simulations were performed using molecular dynamics software GROMACS 2019.6 ${ }^{59}$. For the study of the protein-ligand complex, we only considered the RBD region of the protein (residues 302 to 506) and topology was prepared using AMBER99SB force field ${ }^{60}$. Each complex system was solvated by $\sim 23000$ TIP3P water molecules ${ }^{61}$ in a box of dimension $70 \times 70 \times 180 \AA^{3}$. The physiological concentration $(150 \mathrm{mM})$ of $\mathrm{Na}^{+}$and $\mathrm{Cl}^{-}$ions along with extra $\mathrm{Cl}^{-}$ion were used to neutralize the system.

F. Equilibration and Simulation. Initially each system was energy minimized using steepest descent ${ }^{62}$ method for 10000 steps, followed by heating it to $300 \mathrm{~K}$ in $200 \mathrm{ps}$ using Berendsen thermostat and barostat ${ }^{63}$ with coupling constant of $0.6 \mathrm{ps}$. Restraints of 25 $\mathrm{kcal} / \mathrm{mol} / \AA^{2}$ were applied on heavy atoms during the heating process. Thereafter, equilibration was carried out for $2 \mathrm{~ns}$ at constant temperature $(300 \mathrm{~K})$ and pressure (1 bar) without any restraints using same thermostat and barostat with coupling constants of $0.2 \mathrm{ps}$ each. The last $100 \mathrm{ps}$ of NPT simulation was used to calculate the average volume the same, which was used in the final 5 ns unrestrained NVT equilibration using the Nosé-Hoover ${ }^{64}$ thermostat with coupling constant of 0.2 ps. During the simulation, LINCS algorithm ${ }^{65}$ was used to constrain all the bonds and Particle Mesh Ewald (PME) method ${ }^{66}$ was used for electrostatics. The distance cut-offs for the van der Waals $(\mathrm{vdW})$ and electrostatic long-range interaction was kept at 10 $\AA$. The time step for each simulation was taken to be $2 \mathrm{fs}$.

G. Free energy calculation using metadynamics. The equilibrated Ligand-bound protein structure was initially simulated for $5 \mathrm{~ns}$. If the ligand was found to be bound after $5 \mathrm{~ns}$ simulation, we subjected the system for free energy calculation. To calculate the binding free energy of drugs, well-tempered metadynamics ${ }^{24}$ simula- 
tions were performed after equilibration using native contacts as collective variables (see SI for full description). We performed a long $(\sim 100 \mathrm{~ns})$. metadynamics simulation with a hill height of $0.2 \mathrm{~kJ} / \mathrm{mol}$ and a bias factor of 10 , and hills deposition rate of 2 ps. Gaussian widths for distvec and native contacts were taken to be $0.6 \AA$ and 5, respectively. An upper wall restraint was applied at $45^{\circ}$ on the angle between two vectors $\hat{b}$ and $\vec{d}$. For free-energy calculations, PLUMED $2.6^{67}$ was used along with GROMACS. The system size and run lengths of all the systems are provided in the Table S4 of SI.

\section{ASSOCIATED CONTENT}

\section{SUPPORTING INFORMATION}

DeNovo flow chart, image containing all the selected de novo molecules, table showing list of similar drugs, image showing docking region, table of docking scores for selected drugs, table for docking variations, image of all the selected drug molecules, definition and picture of the collective variables used, table showing system size and other information for all the systems, and free energy surfaces of binding to RBD for all the 55 molecules.

\section{AUTHOR INFORMATION}

Corresponding Author

* Arnab Mukherjee

arnab.mukherjee@iiiserpune.ac.in

Indian Institute of Science Education and Research Pune, Maharashtra, India

Present Addresses

$\uparrow$ Indian Institute of Science Education and Research Kolkata, West Bengal, India

Author Contributions

$\$$ These authors contributed equally.

Funding Sources

This work was partially supported by Department of Biotechnology, India (BT/PR34215/AI/133/22/2019).

\section{ACKNOWLEDGMENT}

Authors acknowledge the support and the resources provided by 'PARAM Brahma Facility' under the National Supercomputing Mission, Government of India at the Indian Institute of Science Education and Research, Pune. Authors also acknowledge help of system administrator Nisha Kurkure. Scientific discussions with Dr. Kausik Chakraborty from IGIB and Samarpita Sen from IISER Kolkata are gratefully acknowledged. RC (fellow id SX-1411075) and VSSA (fellow id: SX-1711011) thank KVPY and AV thanks CSIR for their fellowships.

\section{ABBREVIATIONS}

FES, free energy surface; Cov2, SARS-COV-2. REFERENCES

1. Tang, X. C.; Zhang, J. X.; Zhang, S. Y.; Wang, P.; Fan, X. H.; Li, L. F.; Li, G.; Dong, B. Q.; Liu, W.; Cheung, C. L.; Xu, K. M.; Song, W. J.; Vijaykrishna, D.; Poon, L. L. M.; Peiris, J. S. M.; Smith, G. J. D.; Chen, H.; Guan, Y., Prevalence and genetic diversity of coronaviruses in bats from China. J. Virol. 2006, 80 (15), 7481-7490.

2. Bajusz, D.; Racz, A.; Heberger, K., Why is Tanimoto index an appropriate choice for fingerprint-based similarity calculations? Journal of Cheminformatics 2015, 7.

3. World Health, O. Novel Coronavirus (2019-nCoV): situation report, 19; World Health Organization: Geneva, 2020-0208, 2020.
4. Ge, X. Y.; Li, J. L.; Yang, X. L.; Chmura, A. A.; Zhu, G. J.; Epstein, J. H.; Mazet, J. K.; Hu, B.; Zhang, W.; Peng, C.; Zhang, Y. J.; Luo, C. M.; Tan, B.; Wang, N.; Zhu, Y.; Crameri, G.; Zhang, S. Y.; Wang, L. F.; Daszak, P.; Shi, Z. L., Isolation and characterization of a bat SARS-like coronavirus that uses the ACE2 receptor. Nature 2013, 503 (7477), 535-+.

5. Du, L. Y.; He, Y. X.; Zhou, Y. S.; Liu, S. W.; Zheng, B. J.; Jiang, S. B., The spike protein of SARS-CoV - a target for vaccine and therapeutic development. Nat. Rev. Microbiol. 2009, 7 (3), 226236.

6. Jin, Z.; Du, X.; Xu, Y.; Deng, Y.; Liu, M.; Zhao, Y.; Zhang, B.; Li, X.; Zhang, L.; Peng, C.; Duan, Y.; Yu, J.; Wang, L.; Yang, K.; Liu, F.; Jiang, R.; Yang, X.; You, T.; Liu, X.; Yang, X.; Bai, F.; Liu, H.; Liu, X.; Guddat, L. W.; Xu, W.; Xiao, G.; Qin, C.; Shi, Z.; Jiang, H.; Rao, Z.; Yang, H., Structure of M(pro) from COVID-19 virus and discovery of its inhibitors. Nature 2020.

7. Bzówka, M.; Mitusińska, K.; Raczyńska, A.; Samol, A.; Tuszyński, J.; Góra, A., Molecular Dynamics Simulations Indicate the COVID-19 Mpro Is Not a Viable Target for Small-Molecule Inhibitors Design. bioRxiv 2020, 2020.02.27.968008.

8. Zhou, Y.; Hou, Y.; Shen, J.; Huang, Y.; Martin, W.; Cheng, F., Network-based drug repurposing for novel coronavirus 2019-nCoV/SARS-CoV-2. Cell Discov 2020, 6, 14.

9. Wishart, D. S.; Knox, C.; Guo, A. C.; Cheng, D.; Shrivastava, S.; Tzur, D.; Gautam, B.; Hassanali, M., DrugBank: a knowledgebase for drugs, drug actions and drug targets. Nucleic Acids Res. 2008, 36 (Database issue), D901-6.

10. Shang, J.; Ye, G.; Shi, K.; Wan, Y.; Luo, C.; Aihara, H.; Geng, Q.; Auerbach, A.; Li, F., Structural basis of receptor recognition by SARS-CoV-2. Nature 2020.

11. Wrapp, D.; Wang, N.; Corbett, K. S.; Goldsmith, J. A.; Hsieh, C. L.; Abiona, O.; Graham, B. S.; McLellan, J. S., Cryo-EM structure of the 2019-nCoV spike in the prefusion conformation. Science 2020, 367 (6483), 1260-1263.

12. Yan, R.; Zhang, Y.; Li, Y.; Xia, L.; Guo, Y.; Zhou, Q., Structural basis for the recognition of the SARS-CoV-2 by full-length human ACE2. Science 2020.

13. Walls, A. C.; Park, Y. J.; Tortorici, M. A.; Wall, A.; McGuire, A. T.; Veesler, D., Structure, Function, and Antigenicity of the SARS-CoV-2 Spike Glycoprotein. Cell 2020, 181 (2), 281-292 e6.

14. Letko, M.; Marzi, A.; Munster, V., Functional assessment of cell entry and receptor usage for SARS-CoV-2 and other lineage B betacoronaviruses. Nature Microbiology 2020, 5 (4), 562-+.

15. Smith, J. C.; Smith, M., Repurposing Therapeutics for COVID-19: Supercomputer-Based Docking to the SARS-CoV-2 Viral Spike Protein and Viral Spike Protein-Human ACE2 Interface. 2020.

16. Romulo O., B.; Fabio L. C. C., J.; Wildrimak S., P.; Neiva M. N., O.; Ricardo, R., Interaction of drugs candidates with various SARS-CoV-2 receptors: an in silico study to combat COVID-19. 2020.

17. Ayman, F.; Ping, W.; Mahmoud, A.; Hesham, S., Identification of FDA Approved Drugs Targeting COVID-19 Virus by Structure-Based Drug Repositioning. 2020.

18. Manish, M., Studies on Computational Molecular Interaction Between SARS-CoV-2 Main Protease and Natural Products. 2020.

19. Scior, T.; Bender, A.; Tresadern, G.; Medina-Franco, J. L.; Martinez-Mayorga, K.; Langer, T.; Cuanalo-Contreras, K.; Agrafiotis, D. K., Recognizing Pitfalls in Virtual Screening: A Critical Review. J. Chem. Inf. Model. 2012, 52 (4), 867-881.

20. Guy, R. K.; DiPaola, R. S.; Romanelli, F.; Dutch, R. E., Rapid repurposing of drugs for COVID-19. Science 2020.

21. Wang, L.; Wu, Y. J.; Deng, Y. Q.; Kim, B.; Pierce, L.; Krilov, G.; Lupyan, D.; Robinson, S.; Dahlgren, M. K.; Greenwood, J.; Romero, D. L.; Masse, C.; Knight, J. L.; Steinbrecher, T.; Beuming, T.; Damm, W.; Harder, E.; Sherman, W.; Brewer, M.; Wester, R.; Murcko, M.; Frye, L.; Farid, R.; Lin, T.; Mobley, D. L.; Jorgensen, W. L.; Berne, B. J.; Friesner, R. A.; Abel, R., Accurate and Reliable Prediction of Relative Ligand Binding Potency in 
Prospective Drug Discovery by Way of a Modern Free-Energy Calculation Protocol and Force Field. J. Am. Chem. Soc. 2015, 137 (7), 2695-2703

22. Kirkpatrick, P.; Ellis, C., Chemical space. Nature 2004, 432 (7019), 823-823.

23. Levy, S. B.; Marshall, B., Antibacterial resistance worldwide: causes, challenges and responses. Nat. Med. 2004, 10 (12), S122-S129.

24. Barducci, A.; Bussi, G.; Parrinello, M., Well-tempered metadynamics: A smoothly converging and tunable free-energy method. Phys. Rev. Lett. 2008, 100 (2).

25. Laio, A.; Parrinello, M., Escaping free-energy minima. Proc. Natl. Acad. Sci. U. S. A. 2002, 99 (20), 12562-12566.

26. Willett, P., Similarity-based virtual screening using 2D fingerprints. Drug Discov. Today 2006, 11 (23-24), 1046-1053.

27. Jaccard, P., Etude comparative de la distribution florale dans une portion des Alpes et du Jura. Bull Soc Vaudoise Sci Nat 1901, 37, 547-589.

28. Baldi, P.; Benz, R. W., BLASTing small molecules statistics and extreme statistics of chemical similarity scores. Bioinformatics 2008, 24 (13), I357-I365.

29. Bussi, G.; Laio, A., Using metadynamics to explore complex free-energy landscapes. Nature Reviews Physics 2020, 2 (4), 200-212.

30. Sasikala, W. D.; Mukherjee, A., Intercalation and deintercalation pathway of proflavine through the minor and major grooves of DNA: roles of water and entropy. Phys. Chem. Chem. Phys. 2013, 15 (17), 6446-6455.

31. Capelli, R.; Bochicchio, A.; Piccini, G.; Casasnovas, R.; Carloni, P.; Parrinello, M., Chasing the Full Free Energy Landscape of Neuroreceptor/Ligand Unbinding by Metadynamics Simulations. J. Chem. Theory Comput. 2019, 15 (5), 3354-3361.

32. Wang, J., Fast Identification of Possible Drug Treatment of Coronavirus Disease-19 (COVID-19) through Computational Drug Repurposing Study. J. Chem. Inf. Model. 2020.

33. Sasikala, W. D.; Mukherjee, A., Intercalation and deintercalation pathway of proflavine through the minor and major grooves of DNA: roles of water and entropy. Phys. Chem. Chem. Phys. 2013, 15 (17), 6446-55.

34. Mukherjee, A.; Sasikala, W. D., Drug-DNA Intercalation: From Discovery to the Molecular Mechanism. Dynamics of Proteins and Nucleic Acids 2013, 92, 1-62.

35. Singh, R. K.; Sasikala, W. D.; Mukherjee, A., Molecular Origin of DNA Kinking by Transcription Factors. J Phys Chem B 2015, 119 (35), 11590-6.

36. Pettersen, E. F.; Goddard, T. D.; Huang, C. C.; Couch, G. S.; Greenblatt, D. M.; Meng, E. C.; Ferrin, T. E., UCSF Chimera--a visualization system for exploratory research and analysis. $J$. Comput. Chem. 2004, 25 (13), 1605-12.

37. O'Meara, J. A.; Lemke, C. T.; Godbout, C.; Kukolj, G.; Lagace, L.; Moreau, B.; Thibeault, D.; White, P. W.; Llinas-Brunet, M., Molecular Mechanism by Which a Potent Hepatitis C Virus NS3NS4A Protease Inhibitor Overcomes Emergence of Resistance. $J$. Biol. Chem. 2013, 288 (8), 5673-5681.

38. Alam, N.; Angeli, M. G.; Greenblatt, D. J., Mechanism of in-vitro inhibition of UGT1A1 by paritaprevir. J. Pharm. Pharmacol. 2017, 69 (12), 1794-1801.

39. Jiang, Y. T.; Andrews, S. W.; Condroski, K. R.; Buckman, B.; Serebryany, V.; Wenglowsky, S.; Kennedy, A. L.; Madduru, M. R.; Wang, B.; Lyon, M.; Doherty, G. A.; Woodard, B. T.; Lemieux, C.; Do, M. G.; Zhang, H. L.; Ballard, J.; Vigers, G.; Brandhuber, B. J.; Stengel, P.; Josey, J. A.; Beigelman, L.; Blatt, L.; Seiwert, S. D., Discovery of Danoprevir (ITMN-191/R7227), a Highly Selective and Potent Inhibitor of Hepatitis C Virus (HCV) NS3/4A Protease. $J$. Med. Chem. 2014, 57 (5), 1753-1769.

40. Vella, S.; Floridia, M., Saquinavir - Clinical pharmacology and efficacy. Clin. Pharmacokinet. 1998, 34 (3), 189-201.

41. Putnam, S. D.; Sader, H. S.; Farrell, D. J.; Biedenbach, D. J.; Castanheira, M., Antimicrobial characterisation of solithromycin (CEM-101), a novel fluoroketolide: activity against staphylococci and enterococci. Int. J. Antimicrob. Agents 2011, 37 (1), 39-45.
42. Kobayashi, Y.; Wada, H.; Rossios, C.; Takagi, D.; Higaki, M.; Mikura, S.; Goto, H.; Barnes, P. J.; Ito, K., A novel macrolide solithromycin exerts superior anti-inflammatory effect via NFkappaB inhibition. J. Pharmacol. Exp. Ther. 2013, 345 (1), 76-84.

43. Fernandes, P.; Martens, E.; Bertrand, D.; Pereira, D., The solithromycin journey-It is all in the chemistry. Bioorg. Med. Chem. 2016, 24 (24), 6420-6428.

44. Mantero, M.; Rogliani, P.; Cazzola, M.; Blasi, F.; Di Pasquale, M., Emerging antibacterial and antiviral drugs for treating respiratory tract infections. Expert Opin. Emerg. Drugs 2018, 23 (3), 185-199.

45. Baker, N. C.; Ekins, S.; Williams, A. J.; Tropsha, A., A bibliometric review of drug repurposing. Drug Discov. Today 2018, 23 (3), 661-672.

46. Bonchev, D., Chemical Graph Theory: Introduction and Fundamentals. CRC Press: 2018.

47. Ruddigkeit, L.; van Deursen, R.; Blum, L. C.; Reymond, J. L., Enumeration of 166 Billion Organic Small Molecules in the Chemical Universe Database GDB-17. J. Chem. Inf. Model. 2012, 52 (11), 2864-2875.

48. MacKerell, A. D.; Banavali, N.; Foloppe, N., Development and current status of the CHARMM force field for nucleic acids. Biopolymers 2001, 56 (4), 257-265.

49. Frenkel, D.; Smit, B., Understanding Moleccular Simulation: From Algorithms to Applications. Academic Press Inc.: 6277 Sea Harbor Drive Orlando, FLUnited States, 1996.

50. Case, D. A.; Ben-Shalom, I. Y.; Brozell, S. R.; Cerutti, D. S.; III, T. E. C.; Cruzeiro, V. W. D.; Darden, T. A.; Duke, R. E.; Ghoreishi, D.; Gilson, M. K.; Gohlke, H.; Goetz, A. W.; Greene, D.; Harris, R.; Homeyer, N.; Izadi, S.; Kovalenko, A.; Kurtzman, T.; Lee, T. S.; LeGrand, S.; Li, P.; Lin, C.; Liu, J.; Luchko, T.; Luo, R.; Mermelstein, D. J.; Merz, K. M.; Miao, Y.; Monard, G.; Nguyen, C.; Nguyen, H.; Omelyan, I.; Onufriev, A.; Pan, F.; Qi, R.; Roe, D. R.; Roitberg, A.; Sagui, C.; Schott-Verdugo, S.; Shen, J.; Simmerling, C. L.; Smith, J.; Salomon-Ferrer, R.; Swails, J.; Walker, R. C.; Wang, J.; Wei, H.; Wolf, R. M.; Wu, X.; Xiao, L.; York, D. M.; Kollman, P. A. AMBER 2018, University of California, San Francisco, 2018.

51. Eswar, N.; Webb, B.; Marti-Renom, M. A.; Madhusudhan, M. S.; Eramian, D.; Shen, M. Y.; Pieper, U.; Sali, A., Comparative protein structure modeling using Modeller. Curr Protoc Bioinformatics 2006, Chapter 5, Unit-5 6.

52. Godden, J. W.; Xue, L.; Bajorath, J., Combinatorial preferences affect molecular similarity/diversity calculations using binary fingerprints and Tanimoto coefficients. J. Chem. Inf. Comput. Sci. 2000, 40 (1), 163-166.

53. Goodsell, D. S.; Morris, G. M.; Olson, A. J., Automated docking of flexible ligands: Applications of AutoDock. J. Mol. Recognit. 1996, 9 (1), 1-5.

54. O'Boyle, N. M.; Banck, M.; James, C. A.; Morley, C.; Vandermeersch, T.; Hutchison, G. R., Open Babel: An open chemical toolbox. J Cheminform 2011, 3, 33.

55. Frisch, M. J.; Trucks, G. W.; Schlegel, H. B.; Scuseria, G. E.; Robb, M. A.; Cheeseman, J. R.; Scalmani, G.; Barone, V.; Mennucci, B.; Petersson, G. A.; Nakatsuji, H.; Caricato, M.; Li, X.; Hratchian, H. P.; Izmaylov, A. F.; Bloino, J.; Zheng, G.; Sonnenberg, J. L.; Hada, M.; Ehara, M.; Toyota, K.; Fukuda, R.; Hasegawa, J.; Ishida, M.; Nakajima, T.; Honda, Y.; Kitao, O.; Nakai, H.; Vreven, T.; Jr., J. A. M.; Peralta, J. E.; Ogliaro, F.; Bearpark, M.; Heyd, J. J.; Brothers, E.; Kudin, K. N.; Staroverov, V. N.; Kobayashi, R.; Normand, J.; Raghavachari, K.; Rendell, A.; Burant, J. C.; Iyengar, S. S.; Tomasi, J.; Cossi, M.; Rega, N.; Millam, J. M.; Klene, M.; Knox, J. E.; Cross, J. B.; Bakken, V.; Adamo, C.; Jaramillo, J.; Gomperts, R.; Stratmann, R. E.; Yazyev, O.; Austin, A. J.; Cammi, R.; Pomelli, C.; Ochterski, J. W.; Martin, R. L.; Morokuma, K.; Zakrzewski, V. G.; Voth, G. A.; Salvador, P.; Dannenberg, J. J.; Dapprich, S.; Daniels, A. D.; Farkas, O.; Foresman, J. B.; Ortiz, J. V.; Cioslowski, J.; Fox, D. J. Gaussian 09, Revision A.1, 2009.

56. Wang, J. M.; Wang, W.; Kollman, P. A.; Case, D. A., Automatic atom type and bond type perception in molecular mechanical calculations. J. Mol. Graph. Model. 2006, 25 (2), $247-$ 260 . 
57. Wang, J. M.; Wolf, R. M.; Caldwell, J. W.; Kollman, P. A.; Case, D. A., Development and testing of a general amber force field. J. Comput. Chem. 2004, 25 (9), 1157-1174.

58. Shen, M. Y.; Sali, A., Statistical potential for assessment and prediction of protein structures. Protein Sci. 2006, 15 (11), 250724.

59. Abraham, M. J.; Spoel, D. v. d.; Lindahl, E.; Hess, B.; team, t. G. d. GROMACS User Manual version 2019 http://www.gromacs.org, 2019.

60. Hornak, V.; Abel, R.; Okur, A.; Strockbine, B.; Roitberg, A.; Simmerling, C., Comparison of multiple amber force fields and development of improved protein backbone parameters. ProteinsStructure Function and Bioinformatics 2006, 65 (3), 712-725.

61. Jorgensen, W. L.; Chandrasekhar, J.; Madura, J. D.; Impey, R. W.; Klein, M. L., Comparison of Simple Potential Functions for Simulating Liquid Water. J. Chem. Phys. 1983, 79 (2), 926-935.
62. Press, W. H. T.; Teukolsky, S. A.; Vetterling, W. T.; Flannery, B. P., Numerical Recipes: The Art of Scientific Computing. CAMBRIDGE UNIVERSITY PRESS: 2007.

63. Berendsen, H. J. C.; Postma, J. P. M.; Vangunsteren, W. F.; Dinola, A.; Haak, J. R., Molecular-Dynamics with Coupling to an External Bath. J. Chem. Phys. 1984, 81 (8), 3684-3690.

64. Nose, S., A Molecular-Dynamics Method for Simulations in the Canonical Ensemble. Mol. Phys. 1984, 52 (2), 255-268.

65. Hess, B.; Bekker, H.; Berendsen, H. J. C.; Fraaije, J. G. E. M., LINCS: A linear constraint solver for molecular simulations. $J$. Comput. Chem. 1997, 18 (12), 1463-1472.

66. Darden, T.; York, D.; Pedersen, L., Particle Mesh Ewald an N.Log(N) Method for Ewald Sums in Large Systems. J. Chem. Phys. 1993, 98 (12), 10089-10092.

67. Tribello, G. A.; Bonomi, M.; Branduardi, D.; Camilloni, C.; Bussi, G., PLUMED 2: New feathers for an old bird. Comput. Phys. Commun. 2014, 185 (2), 604-613. 
TOC

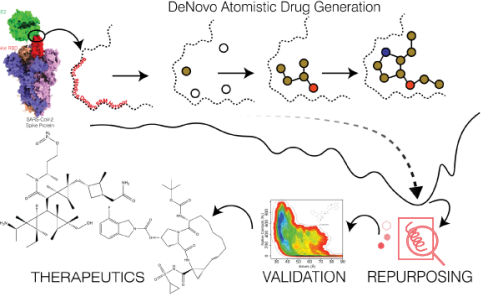


Supporting information for

Generate, repurpose, validate: a receptor-mediated atom-byatom drug generation for SARS-Cov-2 spike protein and similarity-mapped drug repurposing for COVID-19 with rigorous free energy validation using well-tempered metadynamics

Rituparno Chowdhury ${ }^{\dagger}$, Venkata Sai Sreyas Adury ${ }^{\ddagger}$, Amal Vijay $\$$, Reman K. Singh ${ }^{\ddagger}$, and Arnab Mukherjee*

Indian Institute of Science Education and Research Pune, Maharashtra, India

A. DE NOVO PROGRAM FLOWCHART.

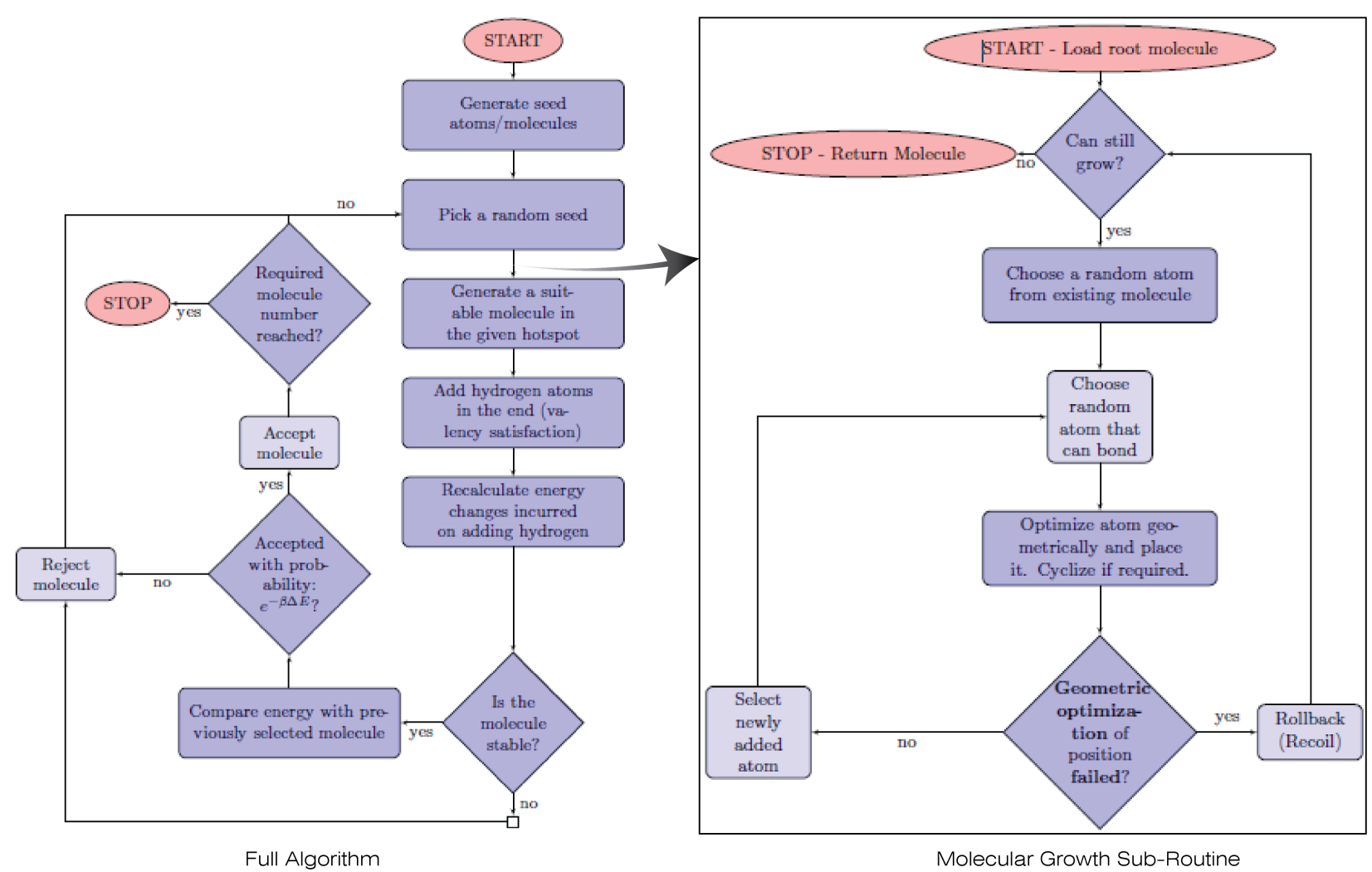

Figure S1. The algorithmic flowchart of de novo drug generation protocol left panel) and the molecular growth subroutine (right panel). 

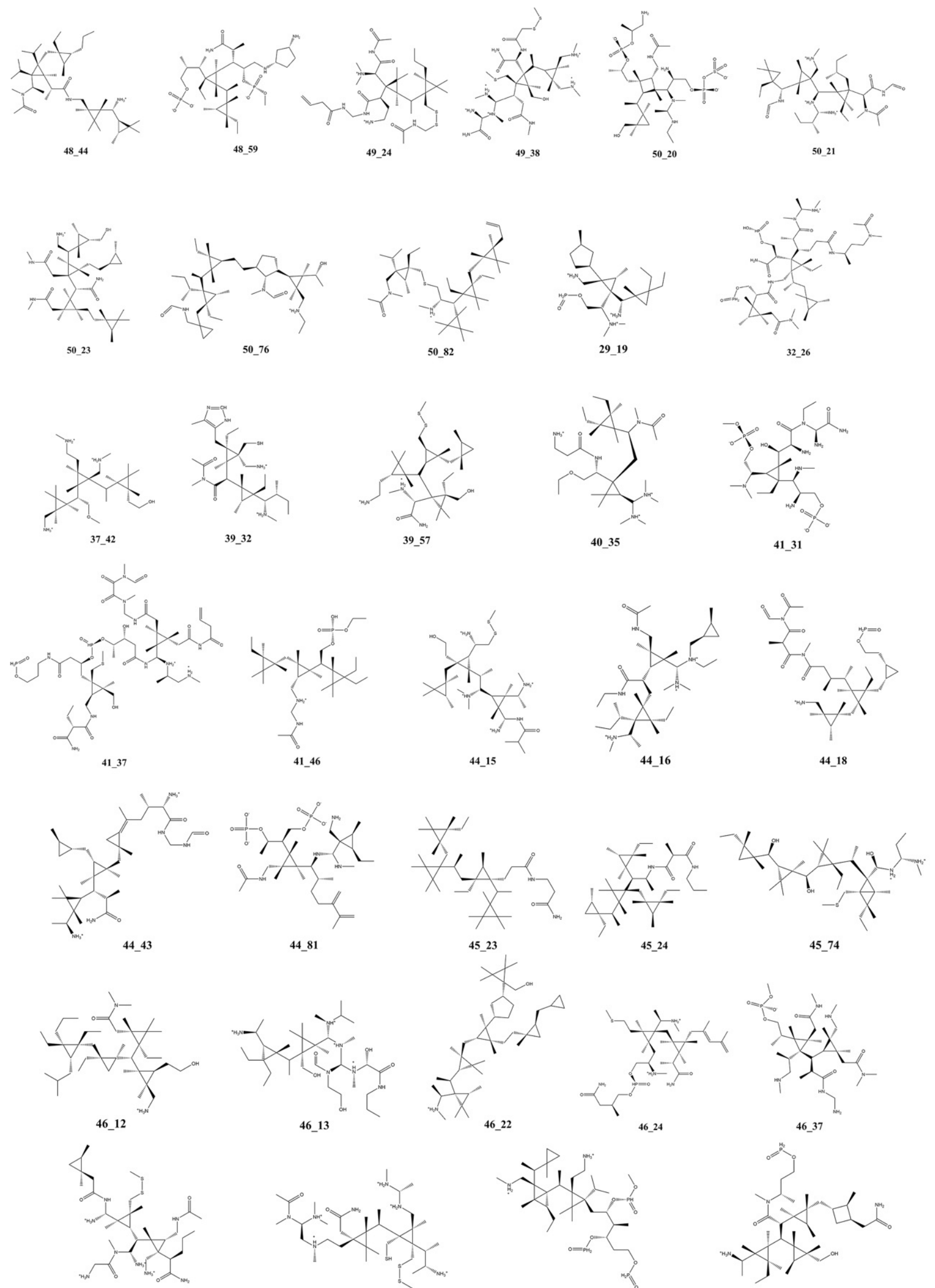

47_11
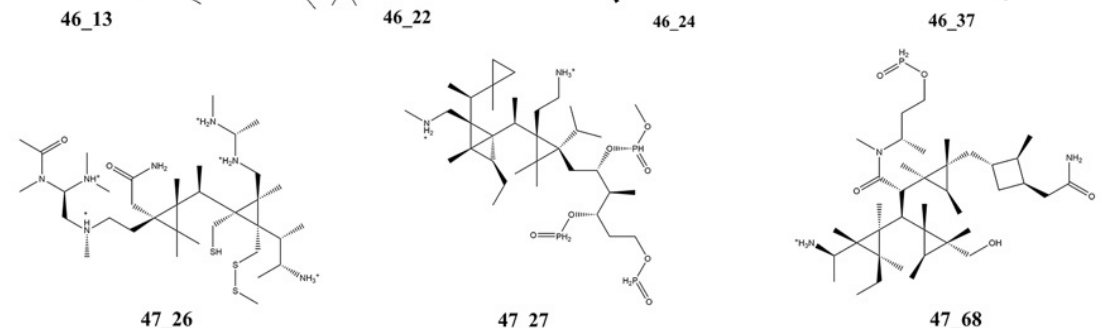

Figure S2. The 35 de novo molecules that made the energy cutoff criteria discussed in the method. Binding free energy of all these molecules were calculated using all-atom explicit water simulations using metadynamics ${ }^{1}$. 


\section{B. TABLE OF SIMILAR DRUG MOLECULES}

Table S1. List of similar drug molecules retrieved from DrugBank ${ }^{2}$ based on Tanimoto coefficient cutoff of 0.4 for each of the chosen de novo molecules. All narcotics, anesthetics, illicit substances, anti-depressants, schizophrenia/Alzheimer's/Parkinson's related hyper specific molecules, contrasting agents, neuromuscular blocking agents, immune-depressants, and pain relievers have been discarded due to their biochemical and pharmacological irrelevance to this problem. These rejects have been marked in red, all others that were acceptable were passed on to docking to RBD and are marked in blue. The docking score and similarity score to the respective de novo molecules are shown in bracket. For the rejected molecules, only similarity score is shown.

\begin{tabular}{|c|c|c|}
\hline $\begin{array}{l}\text { SR. } \\
\text { No. }\end{array}$ & $\begin{array}{l}\text { DeNovo } \\
\text { Id }\end{array}$ & Similar Drugs with DrugBank Id with similarity and docking scores \\
\hline 1 & $29 \_19$ & $\begin{array}{l}\text { DB06200(0.456,-6), DB04878(0.449,-5.6), DB09031(0.444,-5.5), DB01337(0.432), DB01339(0.432), } \\
\text { DB00657(0.422), DB11807(0.422), DB00728(0.418,-6.8), DB05513(0.416), DB11860(0.407,-7.4), } \\
\text { DB00419(0.402), DB14872(0.402), DB04834(0.401,-6) }\end{array}$ \\
\hline 2 & $32 \_26$ & $\begin{array}{l}\text { DB06614(0.557,-5.6), DB11682(0.522,-6.6), DB15286(0.5), DB00312(0.489), DB08873(0.483,-6.1), } \\
\text { DB11860(0.479,-7.4), DB00786(0.478,-6.5), DB06345(0.478,-7.6), DB06293(0.471,-5.8), } \\
\text { DB01351(0.468), DB01353(0.468), DB06335(0.463,-5.9), DB00418(0.463), DB00237(0.46), } \\
\text { DB00790(0.446,-6.2), DB00599(0.445), DB13253(0.442), DB06573(0.441,-6.1), DB06827(0.437,- } \\
\text { 8.2), DB00306(0.436), DB11762(0.433,-6.2), DB01154(0.429), DB06124(0.429,-6.4), } \\
\text { DB00241(0.426), DB00248(0.419,-6.2), DB14932(0.419,-6.5), DB12613(0.419,-6.7), } \\
\text { DB05541(0.418,-5.5), DB00474(0.417), DB11879(0.414), DB05434(0.413,-7), DB06641(0.413,-6), } \\
\text { DB01627(0.412,-5.9), DB11938(0.41,-7.5), DB00178(0.409,-6.8), DB12416(0.407,-7.3), } \\
\text { DB01216(0.406), DB15412(0.406,-5.8), DB00519(0.405,-6.7), DB04845(0.404,-7.1), } \\
\text { DB06127(0.401), DB01190(0.401,-6) }\end{array}$ \\
\hline 3 & $37 \_42$ & $\begin{array}{l}\text { DB00657(0.5), DB11807(0.5), DB06200(0.47,-6), DB12493(0.465), DB06714(0.463), } \\
\text { DB13012(0.463,-6.2), DB15264(0.452), DB00302(0.443), DB04545(0.438,-4.5), DB12032(0.438), } \\
\text { DB01074(0.426), DB04878(0.424,-5.6), DB00996(0.422), DB00230(0.419), DB05513(0.415), } \\
\text { DB12105(0.409), DB01337(0.408), DB01339(0.408), DB11684(0.407), DB01637(0.404), } \\
\text { DB11860(0.404,-7.4) }\end{array}$ \\
\hline 4 & $39 \_32$ & $\begin{array}{l}\text { DB09421(0.536,-7.6), DB12370(0.507,-7), DB11944(0.503), DB15188(0.5,-6.6), DB11695(0.47), } \\
\text { DB12199(0.461,-7.9), DB06763(0.457,-7.4), DB12631(0.45,-7.5), DB12099(0.448), DB01085(0.447), } \\
\text { DB04896(0.446), DB08918(0.446), DB11720(0.437), DB05521(0.436,-7.9), DB01437(0.433), } \\
\text { DB12833(0.433,-8), DB00357(0.43), DB06471(0.429), DB15299(0.429), DB14870(0.428), } \\
\text { DB06335(0.425,-5.9), DB15091(0.421), DB00561(0.418), DB00490(0.418), DB00248(0.417,-6.2), } \\
\text { DB06448(0.416), DB14813(0.408), DB01618(0.406), DB04872(0.406), DB14854(0.406), } \\
\text { DB05796(0.404), DB00117(0.404), DB09102(0.403), DB06645(0.403), DB14955(0.402), } \\
\text { DB09290(0.4) }\end{array}$ \\
\hline 5 & $39 \_57$ & $\begin{array}{l}\text { DB06335(0.546,-5.9), DB12795(0.471,-6.4), DB11860(0.462,-7.4), DB00786(0.453,-6.5), } \\
\text { DB04878(0.442,-5.6), DB04348(0.438,-8.3), DB08834(0.438), DB00790(0.436,-6.2), } \\
\text { DB04876(0.432,-6.5), DB15286(0.429), DB05541(0.424,-5.5), DB02691(0.42), DB06127(0.411), } \\
\text { DB06614(0.408,-5.6), DB15412(0.408,-5.8), DB06657(0.408), DB11684(0.405), DB00141(0.403), } \\
\text { DB12536(0.403), DB01338(0.403,-7.3), DB12677(0.401,-6), DB01627(0.4,-5.9) }\end{array}$ \\
\hline 6 & $40 \_35$ & $\begin{array}{l}\text { DB00790(0.593,-6.2), DB01337(0.557), DB01339(0.557), DB06124(0.54,-6.4), DB04834(0.539,-6), } \\
\text { DB01338(0.536,-7.3), DB11860(0.528,-7.4), DB00728(0.525,-6.8), DB06335(0.514,-5.9), } \\
\text { DB06614(0.514,-5.6), DB00178(0.512,-6.8), DB08965(0.507), DB00519(0.506,-6.7), } \\
\text { DB13288(0.504), DB00479(0.5,-6.1), DB06696(0.485), DB11684(0.484), DB03567(0.483), } \\
\text { DB01197(0.481), DB12232(0.481,-6.1), DB11682(0.476,-6.6), DB13087(0.476), DB12677(0.474,-6), } \\
\text { DB05645(0.472,-6.5), DB12613(0.47,-6.7), DB14932(0.47,-6.5), DB11762(0.467,-6.2), } \\
\text { DB01627(0.466,-5.9), DB12839(0.463), DB06345(0.456,-7.6), DB15286(0.455), DB06045(0.453), } \\
\text { DB05814(0.453), DB01083(0.452), DB00786(0.45,-6.5), DB11879(0.449), DB01190(0.444,-6), } \\
\text { DB06580(0.442), DB12655(0.441), DB04876(0.441,-6.5), DB09291(0.44), DB06472(0.437), } \\
\text { DB05434(0.435,-7), DB05541(0.434,-5.5), DB12416(0.431,-7.3), DB13106(0.43), DB08873(0.43,- } \\
\text { 6.1), DB11929(0.43,-8.2), DB12287(0.43), DB04878(0.429,-5.6), DB06127(0.429), DB12704(0.428), } \\
\text { DB00141(0.425), DB12536(0.425), DB06354(0.425,-7.9), DB08818(0.424), DB09271(0.424), } \\
\text { DB00584(0.423), DB11876(0.422), DB11781(0.422), DB00899(0.421), DB02691(0.42), } \\
\text { DB12615(0.417), DB15412(0.417,-5.8), DB11797(0.415), DB00517(0.413), DB09292(0.412,-8.4), } \\
\text { DB00207(0.412,-7), DB01340(0.411), DB05633(0.408), DB09026(0.408,-6.5), DB00198(0.407), } \\
\text { DB01232(0.407,-8.7), DB06293(0.407,-5.8), DB11801(0.407), DB06497(0.407,-8.1), } \\
\text { DB13262(0.406), DB06573(0.406,-6.1), DB00708(0.404), DB14783(0.403), DB12795(0.403,-6.4), } \\
\text { DB00747(0.401), DB09300(0.401), DB11315(0.401), DB12086(0.401), DB12178(0.401,-8), } \\
\text { DB04863(0.4) }\end{array}$ \\
\hline 7 & $41 \_31$ & $\begin{array}{l}\text { DB06705(0.52), DB06293(0.503,-5.8), DB06124(0.497,-6.4), DB12677(0.495,-6), DB06614(0.489,- } \\
\text { 5.6), DB05434(0.489,-7), DB00786(0.486,-6.5), DB06827(0.484,-8.2), DB08993(0.48,-8.2), } \\
\text { DB01627(0.48,-5.9), DB01190(0.479,-6), DB08873(0.474,-6.1), DB15286(0.473), DB11801(0.47), }\end{array}$ \\
\hline
\end{tabular}




\begin{tabular}{|c|c|c|}
\hline & & $\begin{array}{l}\text { DB12791(0.47,-6.5), DB12791(0.465,-6.5), DB06497(0.46,-8.1), DB00790(0.456,-6.2), } \\
\text { DB08889(0.455,-7.5), DB12613(0.455,-6.7), DB14911(0.452,-7.5), DB00141(0.452), } \\
\text { DB12536(0.452), DB11784(0.451,-6.7), DB12730(0.45,-6.8), DB11762(0.449,-6.2), DB06335(0.449,- } \\
\text { 5.9), DB15412(0.448,-5.8), DB06045(0.448), DB12615(0.448), DB11879(0.445), DB06573(0.444,- } \\
\text { 6.1), DB12704(0.441), DB09291(0.438), DB11890(0.435,-6.7), DB00207(0.435,-7), DB11471(0.434), } \\
\text { DB00479(0.431,-6.1), DB05645(0.431,-6.5), DB12416(0.43,-7.3), DB13034(0.43,-7.3), } \\
\text { DB14932(0.43,-6.5), DB00337(0.43,-8.7), DB00178(0.428,-6.8), DB06696(0.427), DB15188(0.427,- } \\
\text { 6.6), DB15205(0.425), DB00519(0.424,-6.7), DB01082(0.423), DB11938(0.422,-7.5), } \\
\text { DB04876(0.422,-6.5), DB14941(0.422), DB11929(0.421,-8.2), DB12538(0.421,-8.7), DB01590(0.42), } \\
\text { DB06641(0.42,-6), DB00864(0.419), DB13067(0.418), DB00877(0.418,-7.9), DB11797(0.418), } \\
\text { DB00558(0.416) DB12508(0.416,-8.4), DB12279(0.416), DB06233(0.415,-7.6), DB11273(0.415), } \\
\text { DB11274(0.415,-7.4), DB11275(0.415,-7.8), DB06478(0.412), DB05633(0.412), DB13345(0.411), } \\
\text { DB00224(0.411,-7.5), DB14760(0.411,-8.4), DB11781(0.411), DB11586(0.411,-7.3), } \\
\text { DB00305(0.409), DB12989(0.407), DB05541(0.407,-5.5), DB00320(0.407), DB12232(0.406,-6.1), } \\
\text { DB11512(0.406), DB08818(0.405), DB11682(0.405,-6.6), DB09271(0.405), DB01319(0.404), } \\
\text { DB11996(0.404), DB15251(0.404), DB01601(0.403,-7.7), DB12069(0.403,-8), DB04878(0.402,-5.6), } \\
\text { DB09059(0.402), DB11709(0.402), DB00955(0.402), DB06419(0.401), DB15601(0.401), } \\
\text { DB11783(0.401), DB11779(0.401,-9), DB01232(0.40,-8.7) }\end{array}$ \\
\hline 8 & 41_37 & $\begin{array}{l}\text { DB01190(0.483,-6), DB08873(0.48,-6.1), DB01627(0.478,-5.9), DB06573(0.478,-6.1), } \\
\text { DB00760(0.471,-6.2), DB06293(0.463,-5.8), DB06211(0.451,-6.5), DB11586(0.45,-7.3), } \\
\text { DB12791(0.45,-6.5), DB09297(0.45,-9.1), DB12791(0.449,-6.5), DB06233(0.443,-7.6), } \\
\text { DB11929(0.443,-8.2), DB00303(0.441,-7.4), DB12876(0.439,-8.7), DB00503(0.438,-8), } \\
\text { DB15249(0.438,-8), DB06827(0.437,-8.2), DB08993(0.437,-8.2), DB11779(0.436,-9), } \\
\text { DB11273(0.436), DB11274(0.436,-7.4), DB11275(0.436,-7.8), DB11575(0.435,-7.5), } \\
\text { DB12069(0.434,-8), DB12037(0.433,-7.9), DB00248(0.432,-6.2), DB13345(0.432), DB01598(0.432,-- } \\
\text { 5.4), DB12730(0.431,-6.8), DB06335(0.431,-5.9), DB13028(0.428,-6.1), DB12587(0.428,-6.5), } \\
\text { DB05868(0.427,-8.1), DB11808(0.426,-7.3), DB11682(0.426,-6.6), DB01601(0.425,-7.7), } \\
\text { DB00790(0.425,-6.2), DB00320(0.424), DB00696(0.423,-8.7), DB01200(0.423,-7.9), } \\
\text { DB13034(0.422,-7.3), DB12538(0.42,-8.7), DB00739(0.42), DB14911(0.42,-7.5), DB06614(0.419,- } \\
\text { 5.6), DB12677(0.419,-6), DB06124(0.418,-6.4), DB00337(0.418,-8.7), DB09065(0.418), } \\
\text { DB05434(0.418,-7), DB01180(0.418), DB01992(0.418), DB01764(0.417,-7.2), DB00319(0.417,-7.2), } \\
\text { DB14932(0.417,-6.5), DB00948(0.416,-7.5), DB00224(0.416,-7.5), DB14760(0.416,-8.4), } \\
\text { DB05796(0.416), DB08889(0.416,-7.5), DB13879(0.416,-8.3), DB12026(0.416,-7.2), } \\
\text { DB01590(0.415), DB00786(0.415,-6.5), DB09308(0.415,-9), DB12785(0.415), DB01301(0.413), } \\
\text { DB12416(0.413,-7.3), DB00877(0.412,-7.9), DB15284(0.412), DB11961(0.412), DB15040(0.411), } \\
\text { DB06497(0.411,-8.1), DB12279(0.41), DB00256(0.41), DB04845(0.41,-7.1), DB12228(0.41,-8.3), } \\
\text { DB15188(0.41,-6.6), DB06290(0.409,-8.3), DB12691(0.409), DB00864(0.409), DB06604(0.409), } \\
\text { DB12615(0.408), DB00206(0.408), DB11691(0.408), DB11762(0.407,-6.2), DB01089(0.407), } \\
\text { DB12190(0.407), DB12190(0.407), DB12939(0.406), DB15205(0.404), DB12486(0.404), } \\
\text { DB11783(0.402), DB12329(0.402), DB12795(0.402,-6.4), DB00558(0.401), DB00541(0.401), } \\
\text { DB01061(0.4), DB01346(0.4), DB05521(0.4,-7.9), DB13253(0.4), DB01232(0.4,-8.7) }\end{array}$ \\
\hline 9 & $41 \_46$ & $\begin{array}{l}\text { DB06641(0.482,-6), DB09031(0.467,-5.5), DB00312(0.449), DB01351(0.436), DB01353(0.436), } \\
\text { DB00599(0.434), DB00418(0.433), DB13253(0.432), DB00237(0.427), DB01154(0.426), } \\
\text { DB00306(0.413), DB00241(0.411) }\end{array}$ \\
\hline 10 & 44_15 & $\begin{array}{l}\text { DB06641(0.482,-6), DB09031(0.467,-5.5), DB00312(0.449), DB01351(0.436), DB01353(0.436), } \\
\text { DB00599(0.434), DB00418(0.433), DB13253(0.432), DB00237(0.427), DB01154(0.426), } \\
\text { DB00306(0.413), DB00241(0.411) }\end{array}$ \\
\hline 11 & 44_16 & $\begin{array}{l}\text { DB06345(0.557,-7.6), DB00312(0.556), DB08873(0.542,-6.1), DB11879(0.538), DB01351(0.528), } \\
\text { DB01353(0.528), DB05541(0.527,-5.5), DB06614(0.522,-5.6), DB01107(0.52), DB00237(0.519), } \\
\text { DB00418(0.517), DB11682(0.515,-6.6), DB11860(0.513,-7.4), DB05645(0.5,-6.5), DB06335(0.5,- } \\
\text { 5.9), DB00599(0.496), DB15199(0.495,-6.4), DB00593(0.49), DB15286(0.489), DB00306(0.483), } \\
\text { DB00786(0.474,-6.5), DB01154(0.472), DB00241(0.47), DB15601(0.469), DB08958(0.465), } \\
\text { DB06573(0.459,-6.1), DB05434(0.457,-7), DB12833(0.455,-8), DB12839(0.455), DB12613(0.454,- } \\
\text { 6.7), DB06657(0.45), DB00490(0.448), DB00790(0.446,-6.2), DB12692(0.444), DB12991(0.444), } \\
\text { DB13087(0.444), DB13018(0.44), DB01216(0.439), DB06045(0.435), DB13288(0.434), } \\
\text { DB05814(0.433), DB06200(0.433,-6), DB00474(0.432), DB13253(0.431), DB01197(0.429), } \\
\text { DB01252(0.427,-7.9), DB01437(0.424), DB13221(0.421), DB04896(0.419), DB08918(0.419), } \\
\text { DB12444(0.419), DB12951(0.418), DB06124(0.417,-6.4), DB12184(0.417), DB09026(0.407,-6.5), } \\
\text { DB11801(0.406), DB11890(0.405,-6.7), DB00155(0.404), DB00746(0.404), DB11938(0.404,-7.5), } \\
\text { DB00357(0.401), DB11876(0.4) }\end{array}$ \\
\hline 12 & 44_18 & $\begin{array}{l}\text { DB00312(0.56), DB01107(0.556), DB01351(0.532), DB01353(0.532), DB00237(0.523), } \\
\text { DB00418(0.521), DB15286(0.514), DB00593(0.51), DB11860(0.504,-7.4), DB06335(0.493,-5.9), } \\
\text { DB13253(0.492), DB05541(0.491,-5.5), DB00786(0.489,-6.5), DB00306(0.487), DB00241(0.475), } \\
\text { DB11682(0.474,-6.6), DB02691(0.462), DB06200(0.454,-6), DB06614(0.451,-5.6), DB00599(0.45), } \\
\text { DB06293(0.449,-5.8), DB12833(0.448,-8), DB12839(0.446), DB06657(0.441), DB06345(0.44,-7.6), }\end{array}$ \\
\hline
\end{tabular}




\begin{tabular}{|c|c|c|}
\hline & & $\begin{array}{l}\text { DB00474(0.436), DB08873(0.435,-6.1), DB05513(0.432), DB00490(0.431), DB01252(0.431,-7.9), } \\
\text { DB00790(0.43,-6.2), DB01154(0.43), DB01437(0.429), DB01783(0.429), DB05645(0.426,-6.5), } \\
\text { DB13087(0.425), DB11190(0.423), DB04348(0.42,-8.3), DB08834(0.42), DB06274(0.419), } \\
\text { DB09026(0.419,-6.5), DB12712(0.418), DB05814(0.415), DB15412(0.412,-5.8), DB01197(0.411), } \\
\text { DB12951(0.41), DB12613(0.408,-6.7), DB11938(0.407,-7.5), DB12677(0.405,-6), DB01216(0.403), } \\
\text { DB00561(0.403), DB13288(0.402), DB11879(0.402), DB12184(0.4), DB15199(0.4,-6.4) }\end{array}$ \\
\hline 13 & $44 \_43$ & $\begin{array}{l}\text { DB01154(0.563), DB00418(0.546), DB00599(0.526), DB00306(0.513), DB00312(0.491), } \\
\text { DB00241(0.487), DB11825(0.469), DB01351(0.466), DB01353(0.466), DB08873(0.464,-6.1), } \\
\text { DB00237(0.457), DB13253(0.447), DB12513(0.438), DB01216(0.434), DB13221(0.427), } \\
\text { DB12020(0.416), DB15601(0.409), DB00786(0.407,-6.5), DB05541(0.407,-5.5), DB06657(0.402) }\end{array}$ \\
\hline 14 & $44 \_81$ & $\begin{array}{l}\text { DB06614(0.509,-5.6), DB11860(0.459,-7.4), DB15286(0.448), DB06345(0.447,-7.6), } \\
\text { DB06293(0.445,-5.8), DB00786(0.442,-6.5), DB08873(0.441,-6.1), DB06335(0.437,-5.9), } \\
\text { DB11762(0.433,-6.2), DB11682(0.431,-6.6), DB13253(0.424), DB00479(0.42,-6.1), DB14542(0.419,-- } \\
\text { 7.6), DB14631(0.417,-7.4), DB12704(0.416), DB00418(0.416), DB06696(0.408), DB12416(0.407,- } \\
\text { 7.3), DB06124(0.406,-6.4), DB09026(0.405,-6.5), DB11471(0.405), DB04878(0.403,-5.6), } \\
\text { DB06641(0.403,-6) }\end{array}$ \\
\hline 15 & $45 \_23$ & $\begin{array}{l}\text { DB01107(0.7), DB06657(0.6), DB01351(0.59), DB01353(0.59), DB00593(0.571), DB00312(0.57), } \\
\text { DB00237(0.523), DB00418(0.521), DB05541(0.517,-5.5), DB11860(0.516,-7.4), DB00241(0.511), } \\
\text { DB02691(0.495), DB00306(0.479), DB00599(0.479), DB12839(0.477), DB12692(0.475), } \\
\text { DB12991(0.475), DB06200(0.472,-6), DB12951(0.472), DB01783(0.471), DB00746(0.468), } \\
\text { DB06345(0.467,-7.6), DB15199(0.462,-6.4), DB01154(0.451), DB13288(0.449), DB01437(0.449), } \\
\text { DB12521(0.446), DB13253(0.443), DB11190(0.443), DB05513(0.443), DB13099(0.433,-5.1), } \\
\text { DB09357(0.432), DB13087(0.432), DB08873(0.431,-6.1), DB00490(0.425), DB04348(0.425,-8.3), } \\
\text { DB08834(0.425), DB06335(0.424,-5.9), DB15601(0.422), DB00786(0.419,-6.5), DB15286(0.416), } \\
\text { DB12444(0.415), DB01074(0.412), DB09210(0.41), DB01202(0.41), DB11868(0.41), } \\
\text { DB00357(0.407), DB01216(0.403), DB11879(0.402), DB12833(0.4,-8) }\end{array}$ \\
\hline 16 & $45 \_24$ & $\begin{array}{l}\text { DB01107(0.633), DB06345(0.56,-7.6), DB05541(0.559,-5.5), DB11860(0.541,-7.4), DB00593(0.538), } \\
\text { DB01074(0.536), DB13288(0.527), DB00312(0.526), DB06200(0.526,-6), DB01351(0.511), } \\
\text { DB01353(0.511), DB06657(0.506), DB00237(0.5), DB05513(0.5), DB11682(0.5,-6.6), } \\
\text { DB00786(0.492,-6.5), DB01216(0.487), DB00418(0.486), DB06614(0.484,-5.6), DB06335(0.483,- } \\
\text { 5.9), DB00657(0.471), DB11807(0.471), DB11879(0.468), DB08873(0.463,-6.1), DB00306(0.462), } \\
\text { DB15286(0.461), DB00241(0.461), DB15601(0.46), DB05645(0.457,-6.5), DB12951(0.45), } \\
\text { DB00599(0.447), DB06714(0.441), DB01437(0.435), DB05832(0.432,-7.1), DB04876(0.431,-6.5), } \\
\text { DB00746(0.43), DB01197(0.427), DB01202(0.427), DB11868(0.427), DB01154(0.423), } \\
\text { DB12513(0.423), DB12712(0.422), DB01783(0.421), DB08958(0.42), DB13087(0.417), } \\
\text { DB15199(0.416,-6.4), DB00790(0.415,-6.2), DB12839(0.412), DB12833(0.412,-8), DB00141(0.411), } \\
\text { DB12536(0.411), DB01255(0.409), DB05814(0.407), DB05434(0.403,-7), DB13221(0.402), } \\
\text { DB11801(0.402), DB05885(0.402), DB04878(0.4,-5.6), DB09357(0.4), DB12613(0.4,-6.7), } \\
\text { DB06045(0.4) }\end{array}$ \\
\hline 17 & $45 \_74$ & DB04878(0.4,-5.6) \\
\hline 18 & $46 \_12$ & $\begin{array}{l}\text { DB1 1860(0.554,-7.4), DB15264(0.53), DB02691(0.516), DB12032(0.515), DB00230(0.5), } \\
\text { DB00996(0.5), DB13099(0.492,-5.1), DB06335(0.491,-5.9), DB12105(0.486), DB00302(0.478), } \\
\text { DB04348(0.471,-8.3), DB08834(0.471), DB06200(0.459,-6), DB01107(0.458), DB00312(0.457), } \\
\text { DB05541(0.457,-5.5), DB12444(0.457), DB12493(0.456), DB00593(0.455), DB00599(0.454), } \\
\text { DB04878(0.451,-5.6), DB06657(0.443), DB13087(0.438), DB12839(0.433), DB05513(0.431), } \\
\text { DB11825(0.429), DB00149(0.426), DB14187(0.426), DB01351(0.426), DB01353(0.426), } \\
\text { DB00786(0.424,-6.5), DB11684(0.418), DB00657(0.418), DB11807(0.418), DB01154(0.415), } \\
\text { DB00237(0.415), DB00513(0.413), DB00167(0.412), DB12521(0.412), DB05814(0.411), } \\
\text { DB04876(0.411,-6.5), DB00418(0.41), DB15286(0.409), DB13288(0.409), DB15412(0.408,-5.8), } \\
\text { DB06714(0.406), DB01337(0.405), DB01339(0.405), DB09357(0.405), DB00130(0.4) }\end{array}$ \\
\hline 19 & $46 \_13$ & $\begin{array}{l}\text { DB08873(0.492,-6.1), DB06573(0.443,-6.1), DB14941(0.441), DB11682(0.434,-6.6), } \\
\text { DB14075(0.429), DB15286(0.426), DB00790(0.425,-6.2), DB06124(0.424,-6.4), DB06045(0.423), } \\
\text { DB12613(0.422,-6.7), DB14173(0.422), DB04876(0.42,-6.5), DB06293(0.416,-5.8), DB00786(0.412,- } \\
\text { 6.5), DB12416(0.41,-7.3), DB06614(0.41,-5.6), DB13018(0.409), DB06335(0.408,-5.9), } \\
\text { DB15251(0.408), DB06827(0.407,-8.2), DB01601(0.407,-7.7), DB05645(0.405,-6.5), } \\
\text { DB11783(0.405), DB13034(0.4,-7.3) }\end{array}$ \\
\hline 20 & $46 \_22$ & $\begin{array}{l}\text { DB00657(0.714), DB11807(0.714), DB06714(0.612), DB01074(0.554), DB06200(0.516,-6), } \\
\text { DB13012(0.508,-6.2), DB12105(0.5), DB04878(0.494,-5.6), DB12032(0.484), DB11860(0.483,-7.4), } \\
\text { DB00478(0.471), DB04926(0.469), DB05513(0.46), DB11924(0.455), DB15264(0.452), } \\
\text { DB00915(0.451), DB01043(0.451), DB12449(0.446), DB00302(0.443), DB06333(0.43,-6.3), } \\
\text { DB06461(0.43,-6.6), DB11684(0.422), DB00996(0.422), DB01337(0.422), DB01339(0.422), } \\
\text { DB00230(0.419), DB04545(0.419,-4.5), DB13288(0.412), DB00149(0.41), DB12493(0.405), } \\
\text { DB01637(0.404), DB01245(0.4), DB01718(0.4) }\end{array}$ \\
\hline
\end{tabular}




\begin{tabular}{|c|c|c|}
\hline 21 & 46_24 & $\begin{array}{l}\text { DB14631(0.451,-7.4), DB14542(0.444,-7.6), DB05456(0.434), DB11471(0.425), DB12655(0.422), } \\
\text { DB09031(0.419,-5.5), DB01256(0.417), DB14669(0.417), DB09123(0.413), DB06705(0.409), } \\
\text { DB14096(0.407), DB01627(0.406,-5.9), DB06293(0.406,-5.8), DB09091(0.405), DB11921(0.404), } \\
\text { DB11762(0.402,-6.2), DB01190(0.402,-6), DB01590(0.4) }\end{array}$ \\
\hline 22 & 46_37 & $\begin{array}{l}\text { DB06641(0.487,-6), DB00312(0.467), DB09031(0.459,-5.5), DB00599(0.452), DB06293(0.45,-5.8), } \\
\text { DB00418(0.45), DB01154(0.443), DB01351(0.442), DB01353(0.442), DB13253(0.437), } \\
\text { DB15286(0.434), DB00237(0.433), DB06614(0.424,-5.6), DB11860(0.422,-7.4), DB14542(0.42,-7.6), } \\
\text { DB00306(0.419), DB14631(0.409,-7.4), DB00241(0.406), DB15412(0.405,-5.8), DB11848(0.403), } \\
\text { DB06335(0.403,-5.9) }\end{array}$ \\
\hline 23 & 47_11 & $\begin{array}{l}\text { DB05541(0.508,-5.5), DB06335(0.497,-5.9), DB11879(0.496), DB05645(0.486,-6.5), } \\
\text { DB06573(0.468,-6.1), DB12833(0.465,-8), DB13018(0.464), DB08873(0.461,-6.1), DB00786(0.455,-- } \\
\text { 6.5), DB15286(0.451), DB05434(0.449,-7), DB12692(0.448), DB12991(0.448), DB15199(0.447,-6.4), } \\
\text { DB12613(0.447,-6.7), DB00490(0.441), DB00790(0.44,-6.2), DB06124(0.438,-6.4), DB00312(0.43), } \\
\text { DB15601(0.43), DB06657(0.429), DB06127(0.425), DB15412(0.423,-5.8), DB12677(0.422,-6), } \\
\text { DB11709(0.418), DB12795(0.417,-6.4), DB01107(0.416), DB06614(0.415,-5.6), DB12184(0.413), } \\
\text { DB00593(0.412), DB01351(0.407), DB01353(0.407), DB00418(0.407), DB06045(0.403), } \\
\text { DB01597(0.402), DB00237(0.4) }\end{array}$ \\
\hline 24 & $47 \_26$ & $\begin{array}{l}\text { DB08873(0.506,-6.1), DB06573(0.477,-6.1), DB11879(0.465), DB00599(0.46), DB06335(0.459,-5.9), } \\
\text { DB15412(0.458,-5.8), DB00312(0.452), DB01154(0.451), DB13018(0.444), DB15601(0.44), } \\
\text { DB00418(0.438), DB00790(0.432,-6.2), DB05541(0.431,-5.5), DB01351(0.43), DB01353(0.43), } \\
\text { DB05645(0.429,-6.5), DB00474(0.428), DB12795(0.427,-6.4), DB11682(0.425,-6.6), } \\
\text { DB00237(0.422), DB06127(0.418), DB15286(0.417), DB15199(0.416,-6.4), DB06124(0.415,-6.4), } \\
\text { DB05434(0.415,-7), DB00786(0.41,-6.5), DB00306(0.41), DB12613(0.404,-6.7), DB04876(0.4,-6.5), } \\
\text { DB12692(0.4), DB01627(0.4,-5.9), DB12991(0.4) }\end{array}$ \\
\hline 25 & 47_27 & $\begin{array}{l}\text { DB06641(0.515,-6), DB14981(0.48,-5.1), DB14542(0.445,-7.6), DB14631(0.433,-7.4), } \\
\text { DB09031(0.426,-5.5), DB04878(0.418,-5.6), DB11848(0.407) }\end{array}$ \\
\hline 26 & 47 68 & $\begin{array}{l}\text { DB11860(0.579,-7.4), DB06335(0.533,-5.9), DB00786(0.53,-6.5), DB15286(0.521), DB06614(0.511,- } \\
\text { 5.6), DB05541(0.5,-5.5), DB01107(0.477), DB06293(0.476,-5.8), DB00141(0.475), DB12536(0.475), } \\
\text { DB13087(0.471), DB04878(0.47,-5.6), DB05814(0.469), DB00790(0.467,-6.2), DB04876(0.466,-6.5), } \\
\text { DB08873(0.461,-6.1), DB02691(0.458), DB00517(0.458), DB06641(0.457,-6), DB12839(0.456), } \\
\text { DB11684(0.455), DB01197(0.453), DB12677(0.453,-6), DB09357(0.449), DB13288(0.448), } \\
\text { DB12232(0.448,-6.1), DB12704(0.447), DB05645(0.445,-6.5), DB12613(0.445,-6.7), } \\
\text { DB00731(0.442), DB11801(0.442), DB14932(0.441,-6.5), DB01216(0.441), DB00419(0.439), } \\
\text { DB01783(0.439), DB14872(0.439), DB05434(0.438,-7), DB11797(0.438), DB15412(0.438,-5.8), } \\
\text { DB06345(0.437,-7.6), DB00593(0.433), DB00491(0.43), DB04348(0.429,-8.3), DB08834(0.429), } \\
\text { DB06045(0.427), DB11784(0.425,-6.7), DB05805(0.425), DB11938(0.423,-7.5), DB06156(0.423), } \\
\text { DB09292(0.423,-8.4), DB00178(0.422,,-6.8), DB11879(0.421), DB11682(0.421,-6.6), } \\
\text { DB01252(0.419,-7.9), DB11762(0.418,-6.2), DB06354(0.417,-7.9), DB00519(0.417,-6.7), } \\
\text { DB03424(0.417), DB15601(0.416), DB00312(0.415), DB00207(0.414,-7), DB06127(0.414), } \\
\text { DB00907(0.413), DB04896(0.411), DB08918(0.411), DB06657(0.411), DB12013(0.411), } \\
\text { DB00422(0.409), DB06701(0.409), DB09026(0.409,-6.5), DB01337(0.409), DB01339(0.409), } \\
\text { DB06497(0.408,-8.1), DB08824(0.407), DB12712(0.407), DB01437(0.406), DB05137(0.406), } \\
\text { DB01255(0.405), DB06124(0.402,-6.4), DB01627(0.402,-5.9), DB00418(0.402), DB04834(0.401,-6), } \\
\text { DB00479(0.401,-6.1), DB09271(0.401), DB13004(0.401), DB08889(0.401,--7.5), DB04947(0.4), } \\
\text { DB06472(0.4), DB04947(0.4) }\end{array}$ \\
\hline 27 & $48 \_44$ & $\begin{array}{l}\text { DB05541(0.641,-5.5), DB01107(0.627), DB00593(0.595), DB11860(0.57,-7.4), DB11879(0.566), } \\
\text { DB05645(0.563,-6.5), DB06335(0.559,-5.9), DB00786(0.529,-6.5), DB15601(0.527), } \\
\text { DB13288(0.526), DB06657(0.524), DB08873(0.519,-6.1), DB01216(0.512), DB06345(0.51,-7.6), } \\
\text { DB00312(0.51), DB06200(0.506,--6), DB15199(0.505,-6.4), DB13087(0.505), DB05434(0.496,-7), } \\
\text { DB12833(0.492,-8), DB12613(0.492,-6.7), DB00490(0.487), DB01437(0.487), DB05814(0.486), } \\
\text { DB00746(0.483), DB01197(0.482), DB01351(0.48), DB01353(0.48), DB04896(0.479), } \\
\text { DB08918(0.479, DB12839(0.474), DB01074(0.474), DB15286(0.473), DB06614(0.473,-5.6), } \\
\text { DB00418(0.473), DB00790(0.47,-6.2), DB00237(0.47), DB11801(0.468), DB05513(0.462), } \\
\text { DB06045(0.46), DB12184(0.453), DB12444(0.451), DB01255(0.45), DB00599(0.449), } \\
\text { DB01202(0.446), DB11868(0.446), DB05832(0.445,-7.1), DB00357(0.444), DB11682(0.441,-6.6), } \\
\text { DB05885(0.441), DB01252(0.438,-7.9), DB00561(0.438), DB00306(0.436), DB11876(0.436), } \\
\text { DB12951(0.435), DB12712(0.435), DB00657(0.434), DB11807(0.434), DB04876(0.433,-6.5), } \\
\text { DB12521(0.429), DB00141(0.427), DB12536(0.427), DB01154(0.426), DB12513(0.425), } \\
\text { DB01783(0.424), DB06573(0.423,-6.1), DB12692(0.422), DB12991(0.422), DB00241(0.422), } \\
\text { DB00731(0.421), DB02691(0.421), DB09357(0.419), DB12677(0.418,-6), DB11684(0.417), } \\
\text { DB13099(0.417,-5.1), DB09026(0.415,-6.5), DB11938(0.412,-7.5), DB13221(0.406), } \\
\text { DB05521(0.406, -7.9), DB06714(0.405), DB11784(0.405,-6.7), DB11797(0.403), DB00178(0.403,- } \\
\text { 6.8) }\end{array}$ \\
\hline
\end{tabular}




\begin{tabular}{|c|c|c|}
\hline 28 & $48 \_59$ & $\begin{array}{l}\text { DB06705(0.489), DB06641(0.481,-6), DB09031(0.48,-5.5), DB11860(0.475,-7.4), DB13615(0.472), } \\
\text { DB14932(0.443,-6.5), DB00803(0.442), DB06335(0.441,-5.9), DB06293(0.44,-5.8), DB00728(0.44,- } \\
6.8), \text { DB11693(0.439), DB00091(0.438), DB12139(0.438), DB13068(0.438), DB15259(0.438), } \\
\text { DB14631(0.437,-7.4), DB04933(0.433), DB11882(0.431), DB14542(0.43,-7.6), DB02691(0.429), } \\
\text { DB04878(0.426,-5.6), DB06614(0.425,-5.6), DB09114(0.423), DB11283(0.423), DB14099(0.423), } \\
\text { DB14829(0.423), DB00479(0.422,-6.1), DB05456(0.42), DB00786(0.42,-6.5), DB06087(0.419), } \\
\text { DB00207(0.419,-7), DB06696(0.418), DB15286(0.418), DB04834(0.417,-6), DB15412(0.416,-5.8), } \\
\text { DB01337(0.416), DB01339(0.416), DB11471(0.413), DB00517(0.412), DB01111(0.411), } \\
\text { DB14669(0.411), DB06447(0.41), DB09026(0.407,-6.5), DB11797(0.405), DB04660(0.405), } \\
\text { DB00141(0.404), DB12536(0.404), DB11762(0.403,-6.2), DB11332(0.403), DB12655(0.402), } \\
\text { DB05814(0.401), DB12232(0.401,-6.1), DB01627(0.401,-5.9), DB00790(0.4,-6.2), DB11813(0.4) }\end{array}$ \\
\hline 29 & $49 \_24$ & $\begin{array}{l}\text { DB06573(0.586,-6.1), DB08873(0.52,-6.1), DB05645(0.513,-6.5), DB11879(0.503), DB05541(0.483,- } \\
\text { 5.5), DB12613(0.482,-6.7), DB15601(0.478), DB00786(0.473,-6.5), DB12795(0.471,-6.4), } \\
\text { DB05434(0.468,-7), DB06124(0.463,-6.4), DB00312(0.463), DB12677(0.462,-6), DB06045(0.458), } \\
\text { DB13018(0.455), DB11682(0.453,-6.6), DB12833(0.449,-8), DB00418(0.449), DB15286(0.444), } \\
\text { DB11709(0.443), DB01351(0.442), DB01353(0.442), DB06335(0.442,-5.9), DB15188(0.439,-6.6), } \\
\text { DB13090(0.437), DB00237(0.435), DB01282(0.435), DB00490(0.435), DB00790(0.434,-6.2), } \\
\text { DB00599(0.431), DB12692(0.431), DB12991(0.431), DB15199(0.43,-6.4), DB06497(0.429,-8.1), } \\
\text { DB11801(0.428), DB04898(0.427), DB09059(0.425), DB01154(0.425), DB00306(0.423), } \\
\text { DB00474(0.422), DB11938(0.422,-7.5), DB11890(0.422,-6.7), DB06614(0.419,-5.6), } \\
\text { DB15412(0.418,-5.8), DB05885(0.418), DB04842(0.417), DB11784(0.416,-6.7), DB13034(0.415,- } \\
\text { 7.3), DB01601(0.414,-7.7), DB14911(0.413,-7.5), DB01058(0.413), DB11749(0.413), } \\
\text { DB00241(0.413), DB12377(0.413), DB05633(0.411), DB01041(0.411), DB01627(0.41,-5.9), } \\
\text { DB12184(0.41), DB05521(0.409,-7.9), DB15205(0.407), DB06127(0.406), DB09421(0.404,-7.6), } \\
\text { DB00143(0.403), DB12279(0.403), DB09060(0.402), DB09291(0.402) }\end{array}$ \\
\hline 30 & $49 \_38$ & $\begin{array}{l}\text { DB06335(0.548,-5.9), DB06573(0.51,-6.1), DB06124(0.498,-6.4), DB11709(0.493), DB01190(0.492,- } \\
\text { 6), DB08873(0.49,-6.1), DB13034(0.488,-7.3), DB01627(0.487,-5.9), DB12613(0.487,-6.7), } \\
\text { DB12677(0.481,-6), DB00786(0.478,-6.5), DB15286(0.474), DB06614(0.474,-5.6), DB00790(0.464,- } \\
\text { 6.2), DB05645(0.464,-6.5), DB05541(0.461,-5.5), DB06127(0.458), DB04876(0.456,-6.5), } \\
\text { DB11879(0.455), DB00143(0.451), DB15412(0.449,-5.8), DB06045(0.449), DB01282(0.448), } \\
\text { DB12279(0.447), DB14911(0.447,-7.5), DB15188(0.446,-6.6), DB12795(0.446,-6.4), } \\
\text { DB05434(0.443,-7), DB06211(0.441,-6.5), DB12730(0.439,-6.8), DB09059(0.439), DB13018(0.438), } \\
\text { DB00760(0.436,-6.2), DB06827(0.435,-8.2), DB11890(0.43,-6.7), DB00143(0.427), DB11762(0.426,- } \\
\text { 6.2), DB00178(0.423,-6.8), DB06604(0.42), DB05446(0.42), DB06293(0.42,-5.8), DB00519(0.419,- } \\
\text { 6.7), DB06497(0.418,-8.1), DB00303(0.417,-7.4), DB15297(0.416), DB00886(0.414), } \\
\text { DB05633(0.414), DB01598(0.413,-5.4), DB05796(0.412), DB11938(0.41,-7.5), DB04898(0.41), } \\
\text { DB12380(0.409), DB11929(0.408,-8.2), DB15299(0.408), DB13028(0.408,-6.1), DB05521(0.406,- } \\
\text { 7.9), DB01000(0.406), DB09291(0.405), DB12292(0.404), DB12541(0.403), DB15601(0.403), } \\
\text { DB01348(0.403), DB08889(0.4,-7.5) }\end{array}$ \\
\hline 31 & $50 \_20$ & $\begin{array}{l}\text { DB06705(0.459), DB12416(0.429,-7.3), DB12791(0.429,-6.5), DB08873(0.429,-6.1), } \\
\text { DB12704(0.427), DB06614(0.426,-5.6), DB06827(0.42,-8.2), DB12791(0.42,-6.5), DB15286(0.413), } \\
\text { DB11586(0.411,-7.3), DB06641(0.41,-6), DB01346(0.409), DB14941(0.409), DB12730(0.406,-6.8), } \\
\text { DB06124(0.405,-6.4), DB14911(0.402,-7.5), DB01190(0.401,-6) }\end{array}$ \\
\hline 32 & $50 \_21$ & $\begin{array}{l}\text { DB11879(0.716), DB08873(0.655,-6.1), DB05645(0.651,-6.5), DB05541(0.643,-5.5), } \\
\text { DB15601(0.606), DB12613(0.59,-6.7), DB05434(0.583,-7), DB15199(0.583,-6.4), DB00786(0.581,- } \\
6.5), \text { DB06573(0.561,-6.1), DB06045(0.551), DB12692(0.547), DB12991(0.547), DB00790(0.544,- } \\
6.2), \mathrm{DB} 11801(0.543), \mathrm{DB} 06335(0.539,-5.9), \mathrm{DB} 15286(0.537), \mathrm{DB} 11682(0.532,-6.6), \\
\text { DB06614(0.527,-5.6), DB06124(0.525,-6.4), DB11890(0.524,-6.7), DB00490(0.522), } \\
\text { DB12833(0.514,-8), DB11938(0.513,-7.5), DB05633(0.504), DB05832(0.503,-7.1), DB13018(0.496), } \\
\text { DB06345(0.496,-7.6), DB05521(0.495,-7.9), DB05885(0.493), DB12184(0.493), DB06657(0.491), } \\
\text { DB12677(0.486,-6), DB11860(0.484,-7.4), DB00312(0.484), DB01202(0.482), DB11868(0.482), } \\
\text { DB15251(0.48), DB13087(0.48), DB14940(0.477), DB11784(0.476,-6.7), DB04876(0.475,-6.5), } \\
\text { DB11981(0.474), DB11709(0.473), DB00178(0.471,-6.8), DB13247(0.469), DB06497(0.468,-8.1), } \\
\text { DB00418(0.466), DB00519(0.466,-6.7), DB11702(0.465), DB01197(0.463), DB04898(0.461), } \\
\text { DB01351(0.46), DB01353(0.46), DB14911(0.459,-7.5), DB11876(0.458), DB05814(0.456), } \\
\text { DB00722(0.456), DB05155(0.455), DB01058(0.454), DB01058(0.454), DB00237(0.452), } \\
\text { DB12795(0.451,-6.4), DB15188(0.45,-6.6), DB01216(0.45), DB01107(0.448), DB00143(0.448), } \\
\text { DB06293(0.446,-5.8), DB12501(0.446), DB11996(0.446), DB13034(0.441,-7.3), DB06127(0.44), } \\
\text { DB14941(0.439), DB01282(0.438), DB06827(0.437,-8.2), DB00306(0.436), DB00593(0.432), } \\
\text { DB15412(0.429,-5.8), DB01601(0.428,-7.7), DB12377(0.428), DB01255(0.426), DB08958(0.426), } \\
\text { DB12199(0.425,-7.9), DB00241(0.424), DB00599(0.424), DB12128(0.423), DB12239(0.42), } \\
\text { DB09210(0.42), DB09421(0.42,-7.6), DB01154(0.417), DB00731(0.414), DB09059(0.413), } \\
\text { DB13090(0.413), DB00143(0.411), DB00584(0.411), DB01041(0.411), DB05446(0.41), } \\
\text { DB08382(0.409), DB12839(0.408), DB15205(0.407), DB12122(0.407), DB11821(0.406), } \\
\text { DB05426(0.406), DB00380(0.405), DB12013(0.403), DB01627(0.403,-5.9), DB06645(0.403), }\end{array}$ \\
\hline
\end{tabular}




\begin{tabular}{|c|c|c|}
\hline & & $\begin{array}{l}\text { DB13067(0.402), DB09026(0.402,-6.5), DB08889(0.402,-7.5), DB01000(0.402), DB12054(0.402), } \\
\text { DB13288(0.402), DB12492(0.401), DB09060(0.401), DB00895(0.401), DB09291(0.401), } \\
\text { DB01232(0.4,-8.7), DB03424(0.4), DB09286(0.4), DB15460(0.4) }\end{array}$ \\
\hline 33 & $50 \_23$ & $\begin{array}{l}\text { DB13099(0.508,-5.1), DB01107(0.488), DB00312(0.484), DB00599(0.479), DB06657(0.474), } \\
\text { DB00593(0.468), DB01351(0.452), DB01353(0.452), DB00237(0.441), DB01154(0.438), } \\
\text { DB00418(0.433), DB06335(0.425,-5.9), DB05541(0.408,-5.5), DB04348(0.4,-8.3), DB08834(0.4) }\end{array}$ \\
\hline 34 & $50 \_76$ & $\begin{array}{l}\text { DB11860(0.592,-7.4), DB06335(0.564,-5.9), DB04878(0.543,-5.6), DB06614(0.524,-5.6), } \\
\text { DB04876(0.513,-6.5), DB06200(0.513,-6), DB01337(0.482), DB01339(0.482), DB00657(0.479), } \\
\text { DB11807(0.479), DB13288(0.469), DB12287(0.468), DB02691(0.466), DB04545(0.466,-4.5), } \\
\text { DB00491(0.463), DB01338(0.463,-7.3), DB00419(0.458), DB14872(0.458), DB13012(0.452,-6.2), } \\
\text { DB05513(0.45), DB00786(0.448,-6.5), DB00141(0.444), DB04834(0.444,-6), DB12536(0.444), } \\
\text { DB15286(0.444), DB04348(0.443,-8.3), DB08834(0.443), DB05541(0.442,-5.5), DB01074(0.442), } \\
\text { DB06345(0.441,-7.6), DB00728(0.44,-6.8), DB06333(0.44,-6.3), DB06461(0.44,-6.6), } \\
\text { DB12449(0.44), DB11684(0.434), DB13004(0.428), DB03206(0.426), DB05018(0.426), } \\
\text { DB13262(0.422), DB00790(0.42,-6.2), DB00517(0.415), DB12704(0.414), DB00942(0.414), } \\
\text { DB13087(0.411), DB06714(0.411), DB15412(0.41,-5.8), DB01197(0.409), DB00387(0.407), } \\
\text { DB11797(0.407), DB12839(0.406), DB06787(0.405), DB00593(0.404), DB01227(0.403), } \\
\text { DB00376(0.402) }\end{array}$ \\
\hline 35 & $50 \_82$ & $\begin{array}{l}\text { DB06335(0.496,-5.9), DB04876(0.473,-6.5), DB13288(0.431), DB05541(0.421,-5.5), } \\
\text { DB06345(0.408,-7.6), DB15601(0.401) }\end{array}$ \\
\hline
\end{tabular}

\section{DOCKING RELATED INFORMATION.}

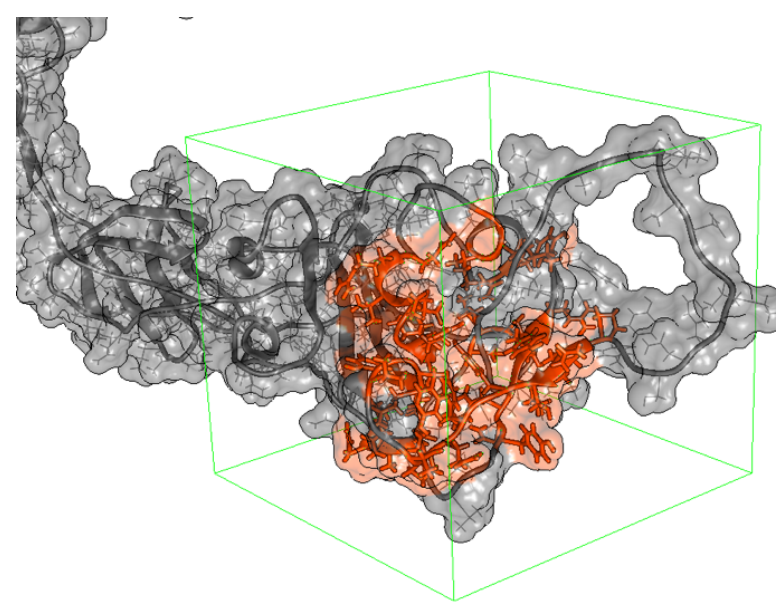

Figure S3. The docking region with the hotspot on SARS-CoV-2 spike RBD that binds to the hACE2 peptidase domain.

Table S2. Docking scores for the final list of drugs.

\begin{tabular}{|l|l|l||l|l|l|}
\hline $\begin{array}{l}\text { DrugBank } \\
\text { ID }\end{array}$ & Drug Name & $\begin{array}{l}\text { Docking } \\
\text { Score } \\
(\mathrm{kcal} / \mathrm{mol})\end{array}$ & $\begin{array}{l}\text { DrugBank } \\
\text { ID }\end{array}$ & Drug Name & $\begin{array}{l}\text { Docking } \\
\text { Score } \\
(\mathrm{kcal} / \mathrm{mol})\end{array}$ \\
\hline DB09297 & Paritaprevir & -9.1 & DB14760 & Narlaprevir & -8.4 \\
\hline DB09308 & Solithromycin & -9.0 & DB04348 & $\begin{array}{l}\text { Taurocholic } \\
\text { acid }\end{array}$ & -8.3 \\
\hline DB11779 & Danoprevir & -9.0 & DB06290 & Simeprevir & -8.3 \\
\hline DB00337 & Pimecrolimus & -8.7 & DB12228 & Telcagepant & -8.3 \\
\hline DB00696 & Ergotamine & -8.7 & DB13879 & Glecaprevir & -8.3 \\
\hline DB01232 & Saquinavir & -8.7 & DB06827 & Viomycin & -8.2 \\
\hline DB12538 & Nepadutant & -8.7 & DB08993 & Enviomycin & -8.2 \\
\hline DB12876 & GS-9256 & -8.7 & DB11929 & Vaniprevir & -8.2 \\
\hline DB09292 & Sacubitril & -8.4 & DB05868 & Ciluprevir & -8.1 \\
\hline DB12508 & E7107 & -8.4 & DB06497 & Aplaviroc & -8.1 \\
\hline
\end{tabular}


Variability in the docking scores: It is important to mention here that several recent studies have used docking for finding repurpose drugs. ${ }^{3-9}$ While docking is good as a relative measure of the strength of binding, it cannot provide an accurate estimate of binding strength. The scores vary depending on the scoring function, grid size, the region selected etc. More importantly, even when same program and other parameters are used, the scores vary based on the molecular representation of the drugs. Table S3 shows that even when using the exact same parameters are used (grid, center, receptor area, etc.) docking scores in AutoDock Vina1.1.2 ${ }^{10}$ vary for the same drug taken directly from the DrugBank ${ }^{2}$ (which does not have nonpolar hydrogen atoms), or after adding nonpolar hydrogens to the same drug obtained from the DrugBank ${ }^{2}$ or after obtaining a 3D optimized configuration of the same drugs using OpenBabel ${ }^{11}$.

Table S3. Docking scores for some drugs of different structures under different levels of hydrogenation and geometric optimization

\begin{tabular}{|l|l|l|l|}
\hline Molecular Code & $\begin{array}{l}\text { Docking Score } \\
\text { polar-H, kcal/mol) }\end{array}$ & $\begin{array}{l}\text { Docking Score (all- } \\
\mathrm{H}, \mathrm{kcal} / \mathrm{mol})\end{array}$ & $\begin{array}{l}\text { Docking Score (all-H + } \\
\text { 3D optimized, kcal/mol) }\end{array}$ \\
\hline DB11929(Vaniprevir) & -9.3 & -7.4 & -8.1 \\
\hline DB14760(Narlaprevir) & -8.7 & -6.5 & -7.6 \\
\hline DB04348(Turocholic Acid) & -8.6 & -7.3 & -8.0 \\
\hline DB01232(Saquinavir) & -8.5 & -7.3 & -8.7 \\
\hline $\begin{array}{l}\text { DB14631(Prednisolone } \\
\text { Phosphate) }\end{array}$ & -8.5 & -7.1 & -7.3 \\
\hline $\begin{array}{l}\text { DB14542(Hydroxycortisone } \\
\text { Phosphate) }\end{array}$ & -8.4 & -7.1 & -7.5 \\
\hline DB00503(Ritonavir) & -8.3 & -7.4 & -8.0 \\
\hline
\end{tabular}


<smiles>C=C(C)c1ccc(CC2CCC(CC3CCC4(CC3)C(C)CC(C(C)C3CCCCC3)C4C)CC2)cc1</smiles>

Aplaviroc

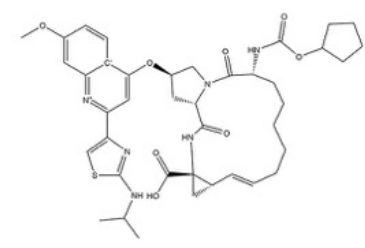

Ciluprevir

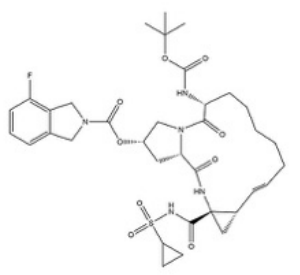

Danoprevir

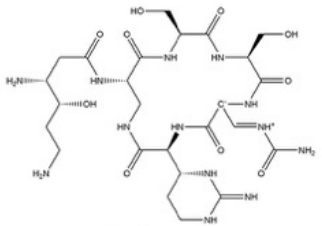

Enviomycin

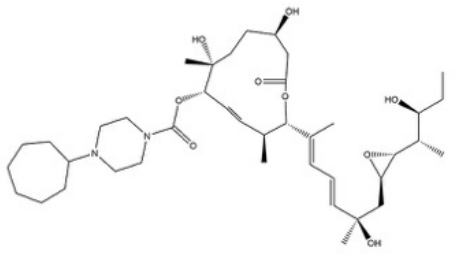

E7107

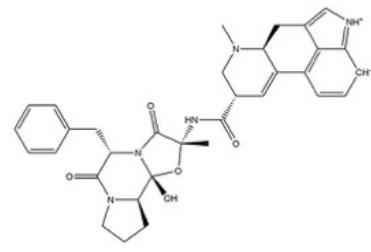

Ergotamine

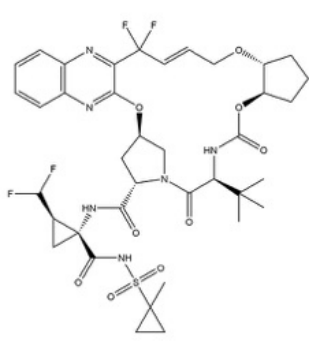

Glecaprevir

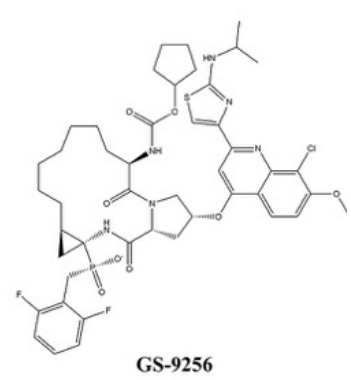

GS-9256

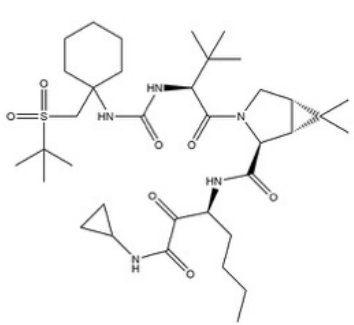

Narlaprevir

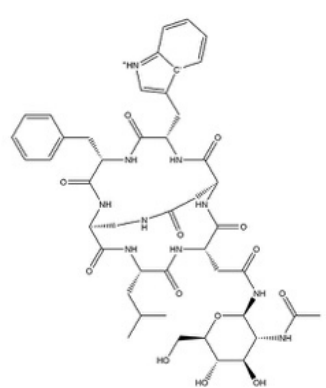

Nepadutant

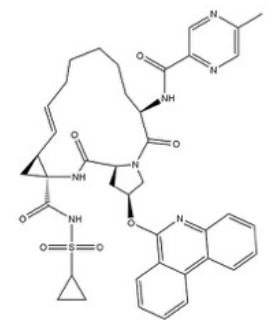

Paritaprevir

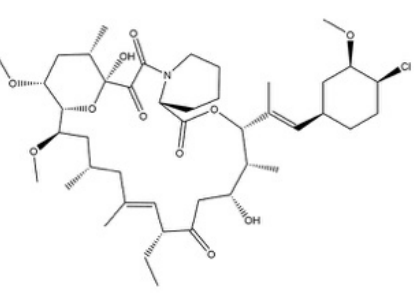

Pimecrolimus

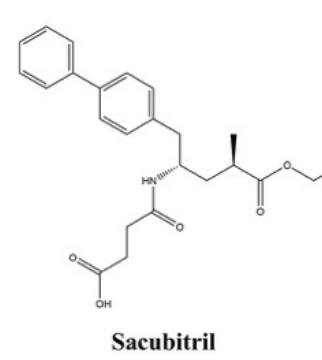

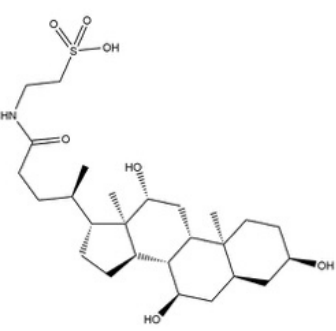

Taurocholic acid

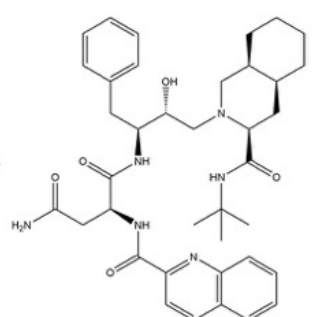

Saquinavir

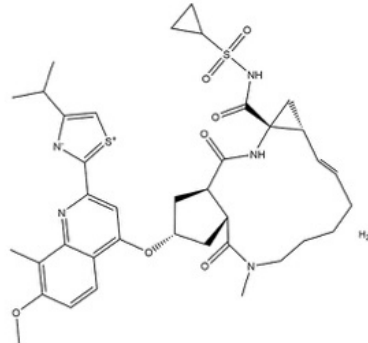

Simeprevir

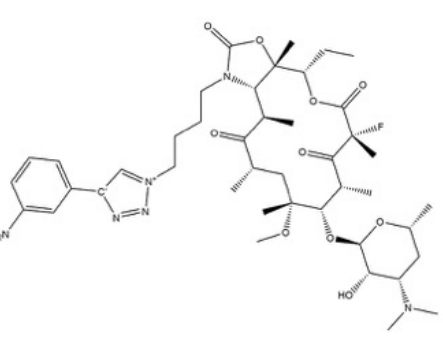

Solithromycin

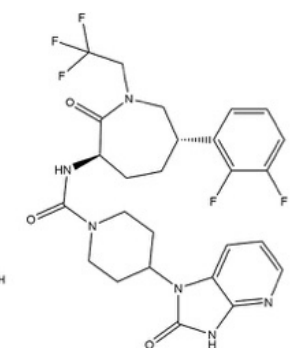

Telcagepant

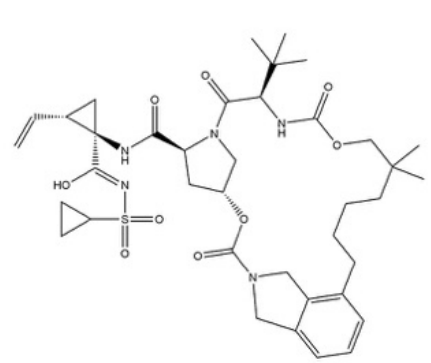

Vaniprevir

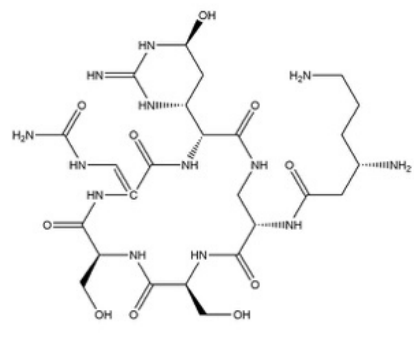

Viomycin

Figure S4. The chemical structures of the drug molecules selected based on the criteria mentioned in the manuscript. Binding free energy of all these molecules were calculated using all-atom explicit water simulations using well-tempered metadynamics ${ }^{1}$. 


\section{FREE ENERGY RELATED INFORMATION}

(1) Definition of Collective Variables. We used two collective variables $\boldsymbol{X}$ and native contact for the well-tempered metadynamics ${ }^{1}$ simulations. We discuss the construction of each of the variables below.

(a) Reaction coordinate distvec is defined by two vectors $\widehat{\boldsymbol{b}}$ and $\overrightarrow{\boldsymbol{d}}$. While $\widehat{\boldsymbol{b}}$ is the unit vector from the COM of residues ARG331, VAL369, TYR370, ALA371, LEU487, SER488, GLU490 to the COM of residues SER323, VAL324, TYR325, VAL375, ILE376, ARG377, GLY378, ASP379, GLU380, VAL381, ARG382, GLN383, GLY390, LYS391, ILE392, TYR395, ASN396, SER412, ASN413, ASN414, LEU415, ASP416, SER417, TYR423, ASN424, TYR425, LEU426, TYR427, ARG428, PRO465, LEU466, GLN467, SER468, TYR469, GLY470, PHE471, GLN472, PRO473, THR474, VAL477, GLY478, TYR479, GLN480, PRO481, TYR482 that lie more toward the hotspot region. $\overrightarrow{\boldsymbol{d}}$ is the vector from the COM of ARG331, VAL369, TYR370, ALA371, LEU487, SER488, GLU490 to the COM of drug molecule. $\boldsymbol{X}=\widehat{\boldsymbol{b}} \cdot \overrightarrow{\boldsymbol{d}}, \boldsymbol{\theta}=$ $\cos ^{-1}(\widehat{b} \cdot \vec{d} /|\boldsymbol{d}|)$. Figure S5 shows a schematic diagram of distvec $(\boldsymbol{X})$.

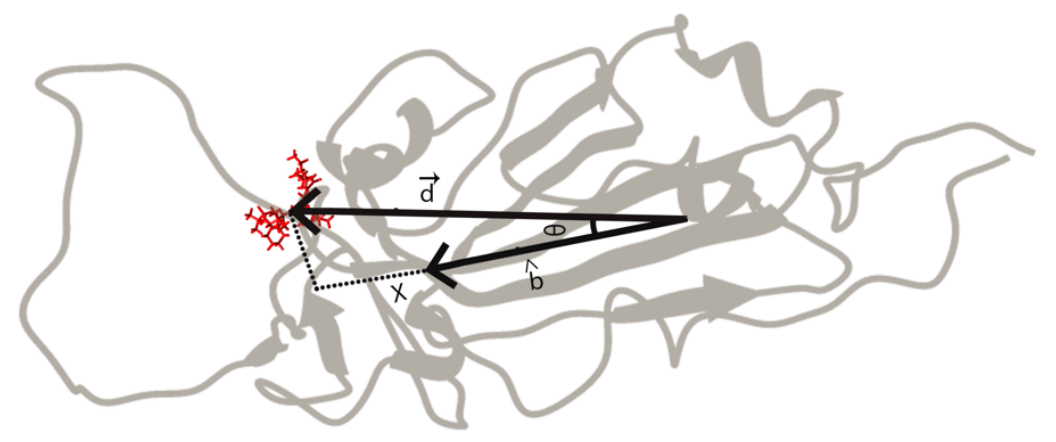

Figure S5. The collective variable distvec $(X)$ and its components are defined in the picture. The protein is shown in grey and ligand is shown in red.

(b) The Native contact $\left(\boldsymbol{N}_{\boldsymbol{c}}\right)$ is defined by the spatial proximity of groups of atoms in the native state. The native contact between one group $(\boldsymbol{g A})$ of atoms to the other group $(\boldsymbol{g B})$ of atoms is defined as, ${ }^{12}$

where, $\boldsymbol{s}_{i j}$ given by,

$$
N_{c}=\sum_{i \in g A} \sum_{i \in g B} s_{i j}
$$

$$
s_{i j}= \begin{cases}1 & \forall r_{i j} \leq 0 \\ \left(1-\left(\frac{r_{i j}}{r_{0}}\right)^{n}\right) /\left(1-\left(\frac{r_{i j}}{r_{0}}\right)^{m}\right) & \forall r_{i j}>0\end{cases}
$$

and $\boldsymbol{r}_{\boldsymbol{i j}}=\left|\boldsymbol{r}_{\boldsymbol{i}}-\boldsymbol{r}_{\boldsymbol{j}}\right|-\boldsymbol{d}_{\mathbf{0}}$. The user-defined parameters were chosen to be $n=6, m=0, \boldsymbol{r}_{\mathbf{0}}=5.5 \AA$ and $\boldsymbol{d}_{\mathbf{0}}=0 \AA$. Above equation ensures the variation of $\boldsymbol{s}_{\boldsymbol{i}}$ is continuous and differentiable. Also, the cut off value being large allows $\boldsymbol{N}_{\boldsymbol{c}}$ to be higher than in the native state if more atoms come close. The heavy atoms of the ligand constitute $\boldsymbol{g A}$ while the heavy atoms of part of the protein (residues 376-377, 379, 382-383,389-392,394-396, 427-430, 440-470, 475, 477, 479) constitute $\boldsymbol{g B}$. We have chosen the residues of the protein that either belong to the loop (residues 442 to 468 ) or reside within $5.5 \AA$ from the ligand in the bound state. Figure S6 shows the protein's region in yellow and the ligand in orange.

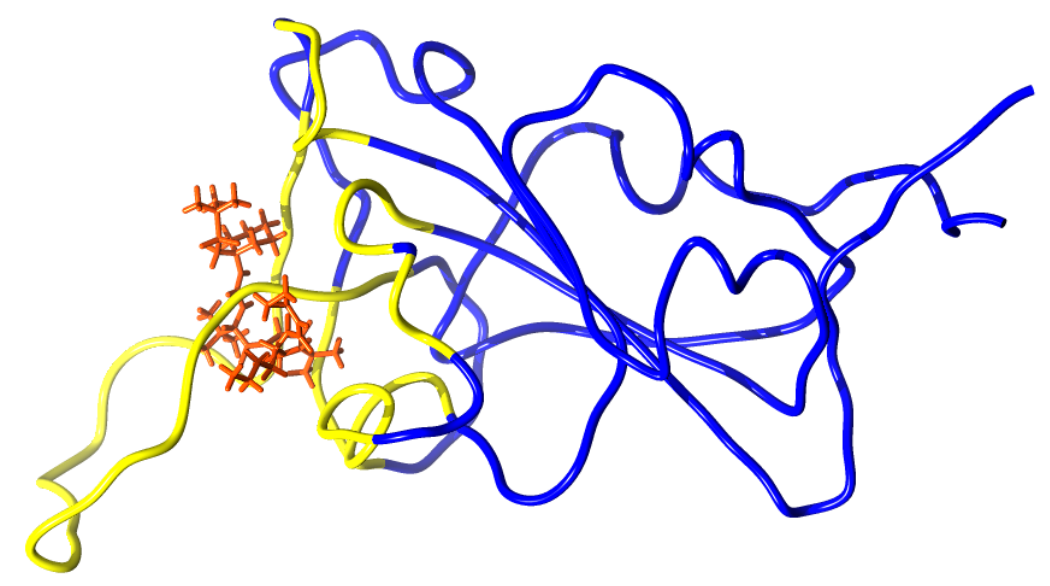

Figure S6. The collective variable native contact is shown. $\boldsymbol{g} \boldsymbol{A}$ (ligand) is shown in orange and $\boldsymbol{g} \boldsymbol{B}$ (part of the protein) is shown in yellow. 
Table S4. The system size and simulation lengths for the metadynamics simulation for all the systems. A general optimization, heating and $5 \mathrm{~ns}$ normal molecular dynamics simulations are applied to all the systems. The metadynamics simulations were not carried out for the unstable system and runtime is denoted for them as "NA".

\begin{tabular}{|c|c|c|c|c|c|}
\hline System & $\begin{array}{l}\text { Runtime } \\
\text { (ns) }\end{array}$ & $\begin{array}{l}\text { System Size } \\
\text { (number of atoms) }\end{array}$ & System & $\begin{array}{l}\text { Runtime } \\
\text { (ns) }\end{array}$ & $\begin{array}{l}\text { System Size } \\
\text { (number of } \\
\text { atoms) }\end{array}$ \\
\hline $\begin{array}{l}\text { hACE2 } \\
\text { (run 1) }\end{array}$ & 234 & 236699 & $\begin{array}{l}\text { hACE2 } \\
\text { (run 2) }\end{array}$ & 51 & 236699 \\
\hline $\begin{array}{l}47 \_68 \\
\text { (run 1) }\end{array}$ & 154 & 86305 & Danoprevir & 236 & 114053 \\
\hline $\begin{array}{l}47 \_68 \\
\text { (run2) }\end{array}$ & 381 & 86305 & Danoprevir & 99 & 114053 \\
\hline $\begin{array}{l}37 \_42 \\
\text { (run } 1\end{array}$ & 41 & 86337 & Solithromycin & 149 & 114129 \\
\hline $\begin{array}{l}37 \quad 42 \\
\text { (run2) }\end{array}$ & 64 & 86337 & Solithromycin & 157 & 114129 \\
\hline $\begin{array}{l}45 \_23 \\
\text { (run 1) }\end{array}$ & 115 & 114066 & Saquinavir & 44 & 86314 \\
\hline $\begin{array}{l}45 \_23 \\
\text { (run 2) }\end{array}$ & 126 & 114066 & $\begin{array}{l}\text { Saquinavir } \\
\text { (run 2) }\end{array}$ & 104 & 86314 \\
\hline $\begin{array}{l}41 \_46 \\
\text { (run 1) }\end{array}$ & 64 & 114089 & Glecaprevir & 166 & 86308 \\
\hline $\begin{array}{l}41 \_46 \\
\text { (run 2) }\end{array}$ & 67 & 114089 & Paritaprevir & 106 & 114087 \\
\hline $49 \_24$ & 48 & 86318 & Ciluprevir & 118 & 86330 \\
\hline $50 \_76$ & 88 & 86329 & Simeprevir & 56 & 114028 \\
\hline $50 \_21$ & 156 & 114066 & Paritaprevir & 48 & 114087 \\
\hline $45 \_24$ & 72 & 86323 & E7107 & 124 & 114121 \\
\hline 44_18 & 109 & 114091 & Ergotamine & 240 & 114037 \\
\hline $50 \_23$ & 75 & 86324 & GS-9256 & 156 & 86284 \\
\hline $50 \_20$ & 30 & 86318 & Enviomycin & 62.9 & 86351 \\
\hline $48 \_44$ & 92 & 86318 & Nepadutant & 53 & 86294 \\
\hline 44_16 & 20 & 114068 & $\begin{array}{l}\text { Vaniprevir } \\
\text { (run 1) }\end{array}$ & 97 & 86309 \\
\hline 47_27 & 63 & 114028 & $\begin{array}{l}\text { Vaniprevir } \\
\text { (run 2) }\end{array}$ & 151 & 86309 \\
\hline $44 \_43$ & 37 & 99557 & Telcagepant & 88 & 86316 \\
\hline $46 \_24$ & 16 & 114066 & Pimecrolimus & 73 & 114056 \\
\hline $39 \_32$ & 14 & 86356 & Narlaprevir & 10.4 & 86376 \\
\hline
\end{tabular}




\begin{tabular}{|l|l|l|l|l|l|}
\hline $46 \_13$ & 24 & 86310 & Sacubitril & 29 & 86338 \\
\hline $46 \_12$ & 13 & 86310 & $\begin{array}{l}\text { Taurocholic } \\
\text { acid }\end{array}$ & 24 & 86352 \\
\hline $29 \_19$ & 14 & 114079 & Aplaviroc & 52 & 86307 \\
\hline $46 \_37$ & 11 & 114066 & Viomycin & 6 & 86309 \\
\hline $47 \_11$ & 18 & 86318 & $49 \_38$ & NA & 86318 \\
\hline $47 \_26$ & 23 & 86305 & $45 \_74$ & NA & 114074 \\
\hline $41 \_37$ & 16 & 86283 & $\begin{array}{l}44 \_15 \\
\text { (run 1) }\end{array}$ & NA & 86298 \\
\hline $50 \_82$ & 14 & 86316 & $\begin{array}{l}44 \_15 \\
\text { (run 2) }\end{array}$ & NA & 86298 \\
\hline $41 \_31$ & 8 & 114039 & $39 \_57$ & NA & 86330 \\
\hline $44 \_81$ & 3 & 86293 & $32 \_26$ & NA & 86300 \\
\hline $46 \_22$ & 18 & 114066 & $40 \_35$ & NA & 86327 \\
\hline $48 \_59$ & 7 & 114066 & & & \\
\hline
\end{tabular}


(2) Free Energy Surfaces. The two-dimensional free energy surfaces of binding of all stable the de novo and drug molecules are shown, except some of. All the plots are made using the software MetadynMiner ${ }^{13}$.
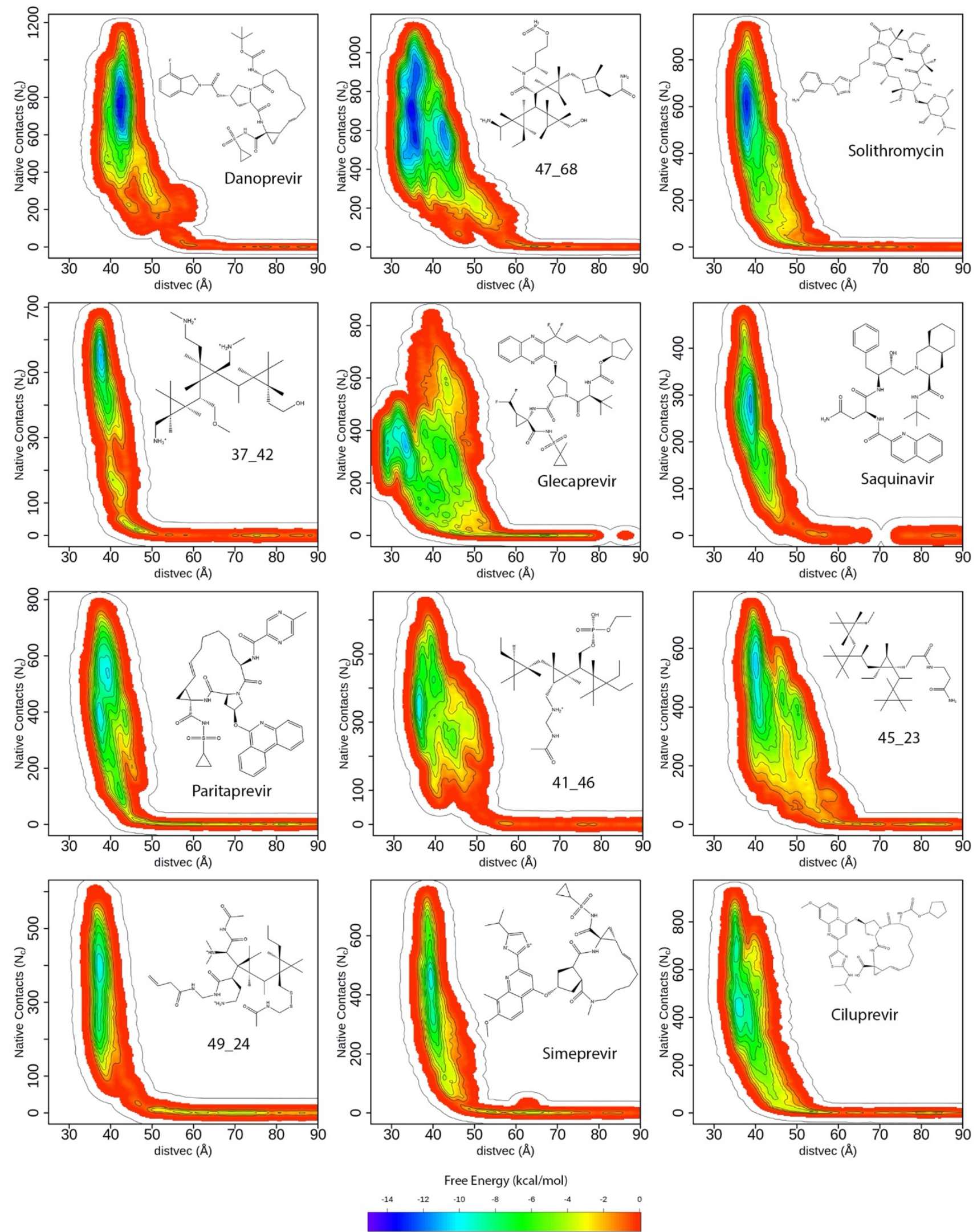

Figure S7. Free Energy surfaces of de novo and drug molecules with free energy stability between $-14.4 \mathrm{kcal} / \mathrm{mol}$ and -9.9 $\mathrm{kcal} / \mathrm{mol}$. The chemical structure of the molecule is shown in the inset of the respective plot. Free energy color bar for this plot is shown. 

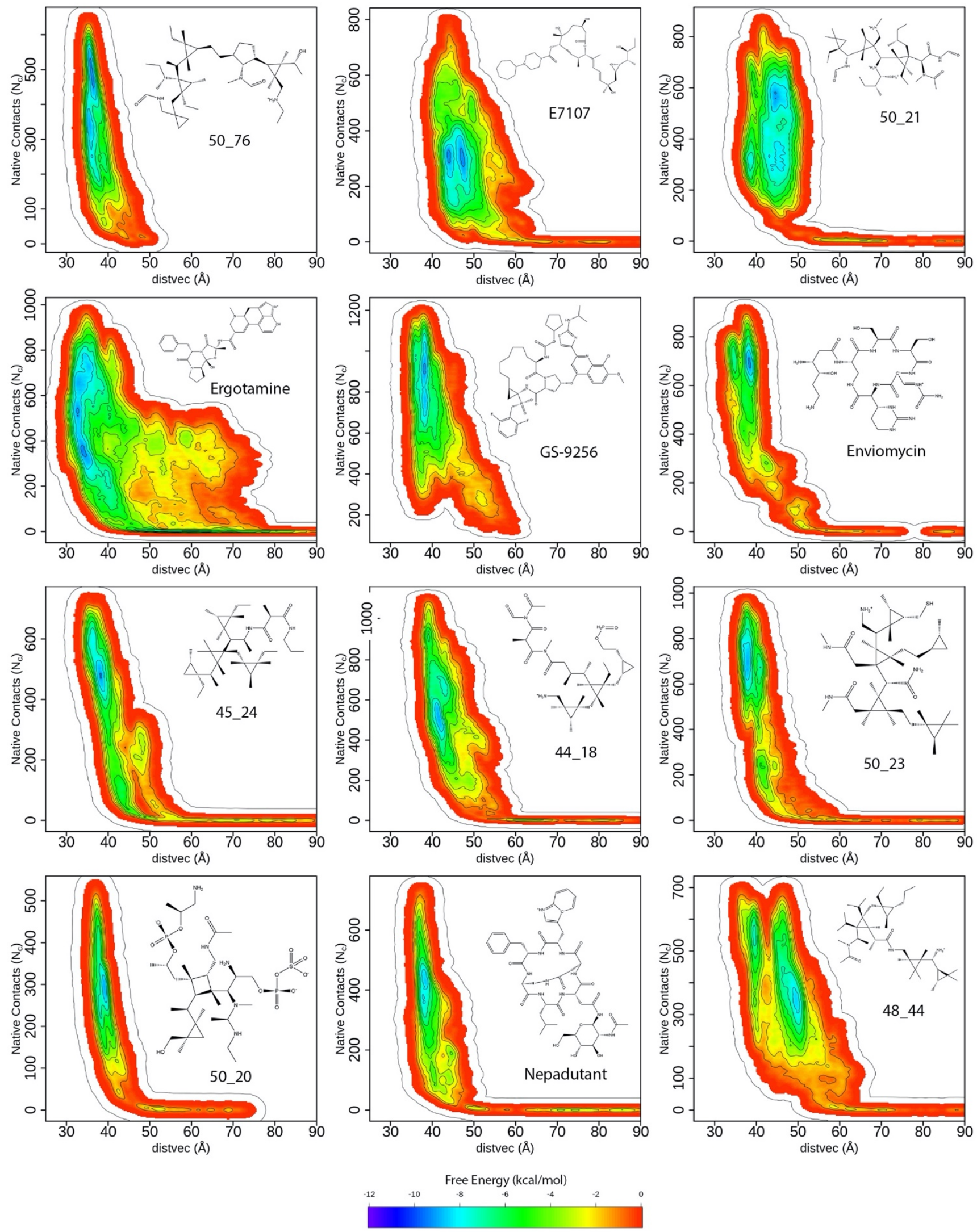

Figure S8. Free Energy surfaces of de novo and drug molecules with free energy stability between $-9.8 \mathrm{kcal} / \mathrm{mol}$ and -8.7 $\mathrm{kcal} / \mathrm{mol}$. The chemical structure of the molecule is shown in the inset of the respective plot. Free energy color bar for this plot is shown. 

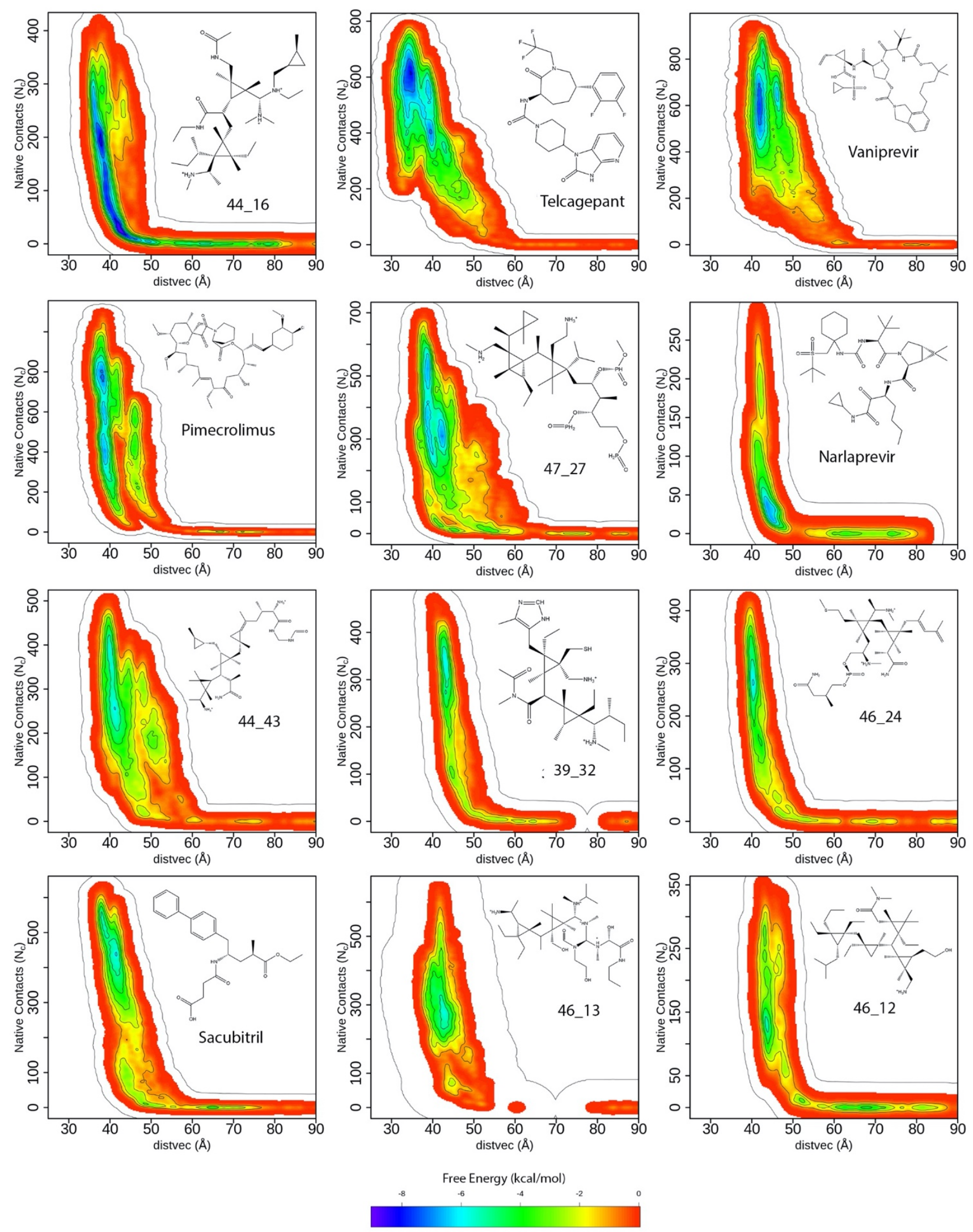

Figure S9. Free Energy surfaces of de novo and drug molecules with free energy stability between $-8.6 \mathrm{kcal} / \mathrm{mol}$ and -5.6 $\mathrm{kcal} / \mathrm{mol}$ (both ends are included). The chemical structure of the molecule is shown in the inset of the respective plot. Free energy color bar for this plot is shown. 

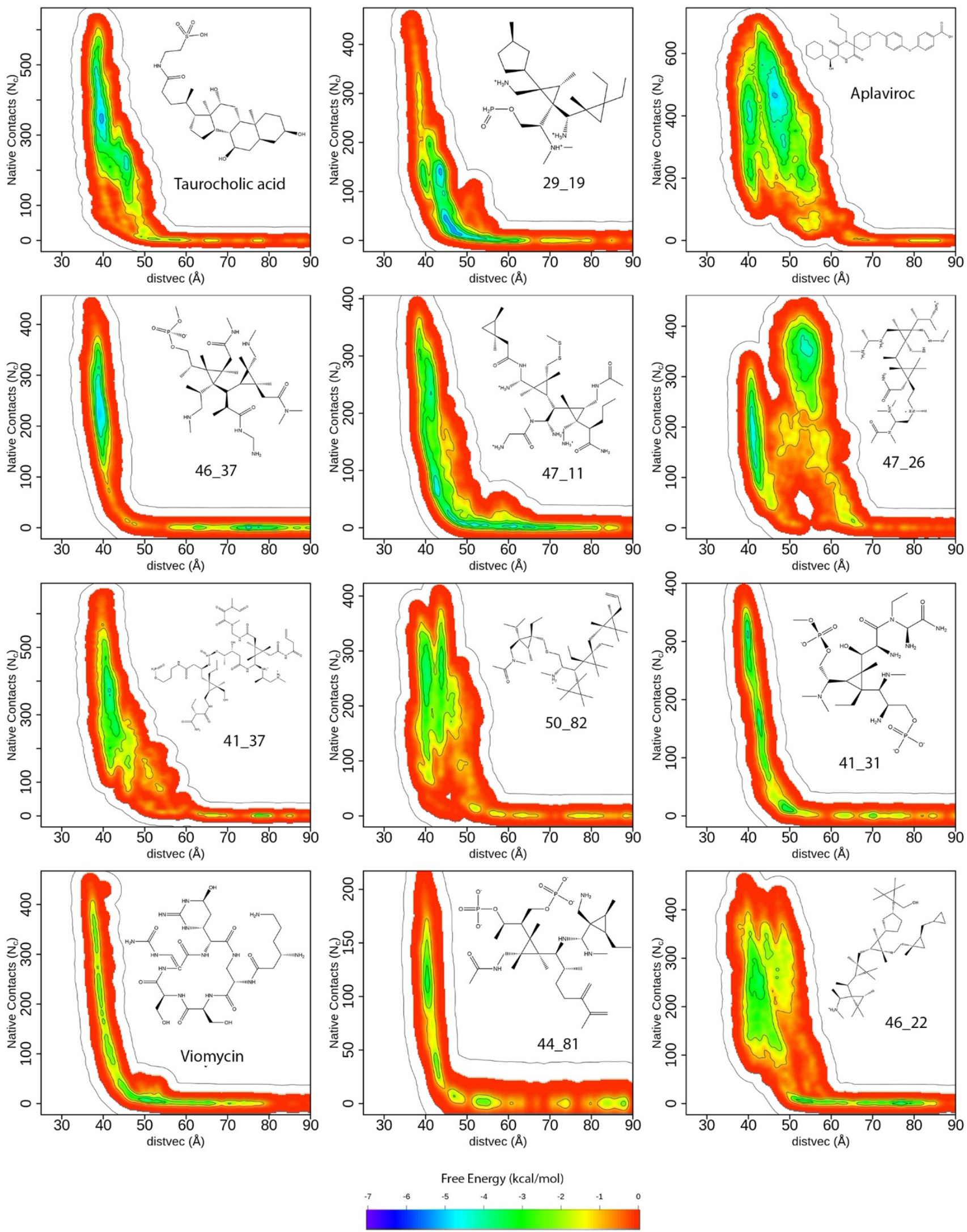

Figure S10. Free Energy surfaces of de novo and drug molecules with free energy stability between $-5.5 \mathrm{kcal} / \mathrm{mol}$ and -3.4 $\mathrm{kcal} / \mathrm{mol}$ (both ends are included). The chemical structure of the molecule is shown in the inset of the respective plot. Free energy color bar for this plot is shown. 


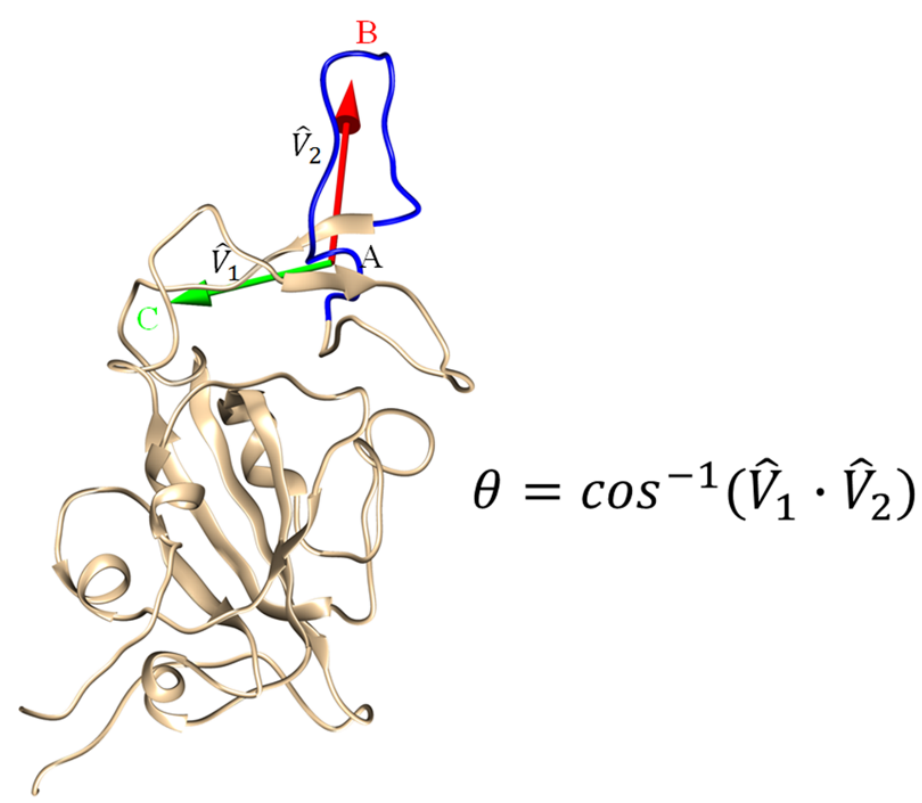

Figure S11. Representation of angle $\theta$ between vectors $\widehat{V}_{1}$ and $\widehat{V}_{2} . \widehat{V}_{1}$ is a vector from the point A to point C, whereas $\widehat{V}_{2}$ goes from point $A$ to point $B$. The point $A$ is the center of mass (COM) of residues 423 to 429 and 465 to 470 . The point $\mathrm{C}$ is the $\mathrm{COM}$ of residues 415 to 422 and 470 to 479 . The point $\mathrm{B}$ is the COM of residues 454 to 459 . Only carbon, nitrogen and oxygen atoms were used to calculate COM calculation.

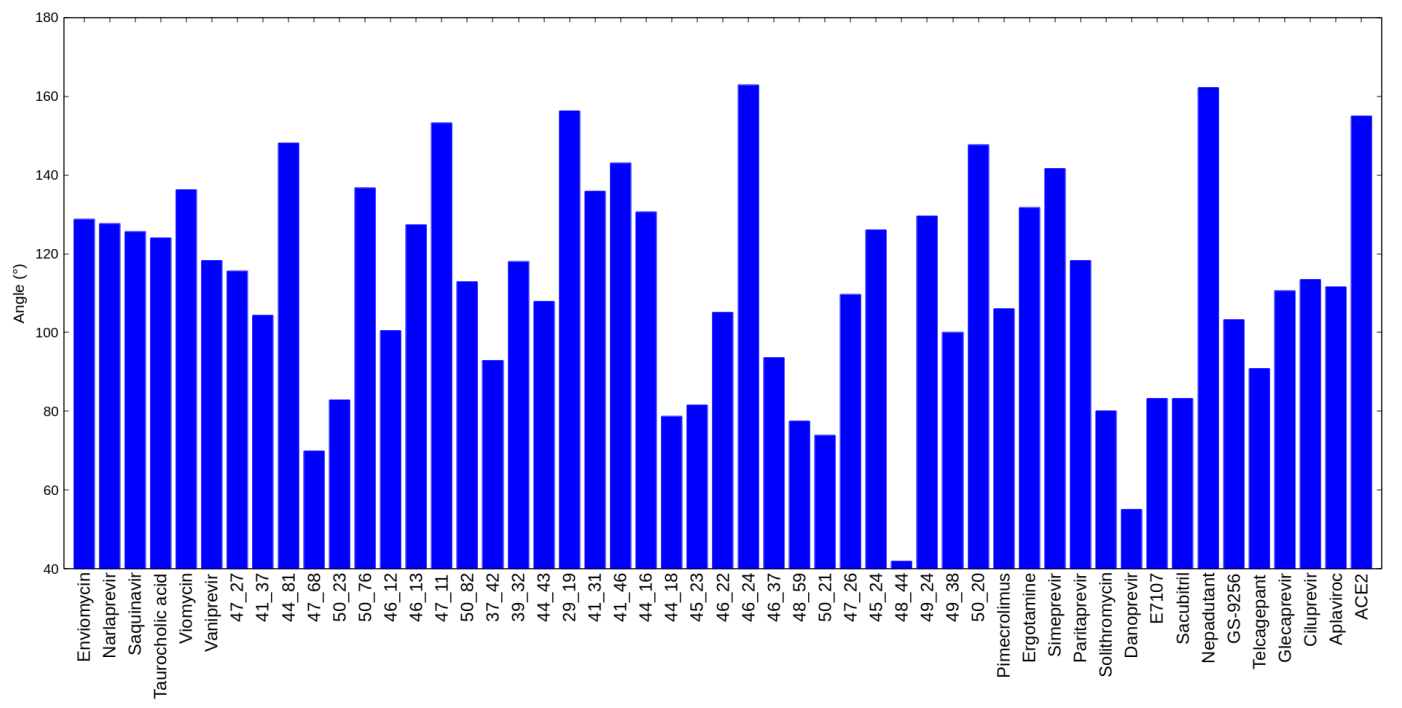

Figure S12. The values of $\theta$ for the most stable ligand bound RBD configurations for all the 35 de novo molecules and 20 drugs. Note that, although the $\theta$ is low for $48 \_44$, the molecule is not encapsulated by the loop.

\section{References}

1. Barducci, A.; Bussi, G.; Parrinello, M., Well-tempered metadynamics: A smoothly converging and tunable free-energy method. Phys. Rev. Lett. 2008, 100 (2).

2. Wishart, D. S.; Knox, C.; Guo, A. C.; Cheng, D.; Shrivastava, S.; Tzur, D.; Gautam, B.; Hassanali, M., DrugBank: a knowledgebase for drugs, drug actions and drug targets. Nucleic Acids Res. 2008, 36 (Database issue), D901-6.

3. Smith, J. C.; Smith, M., Repurposing Therapeutics for COVID-19: Supercomputer-Based Docking to the SARS-CoV-2 Viral Spike Protein and Viral Spike Protein-Human ACE2 Interface. 2020. 
4. Romulo O., B.; Fabio L. C. C., J.; Wildrimak S., P.; Neiva M. N., O.; Ricardo, R., Interaction of drugs candidates with various SARS-CoV-2 receptors: an in silico study to combat COVID-19. 2020.

5. Ayman, F.; Ping, W.; Mahmoud, A.; Hesham, S., Identification of FDA Approved Drugs Targeting COVID-19 Virus by Structure-Based Drug Repositioning. 2020.

6. Micael Davi, L. d. O.; Kelson Mota, T. d. O., Comparative Computational Study of SARS-CoV-2 Receptors Antagonists from Already Approved Drugs. 2020.

7. Manish, M., Studies on Computational Molecular Interaction Between SARS-CoV-2 Main Protease and Natural Products. 2020

8. Thuy, B. T. P.; My, T. T. A.; Hai, N. T. T.; Hieu, L. T.; Hoa, T. T.; Thi Phuong Loan, H.; Triet, N. T.; Anh, T. T. V.; Quy, P. T.; Tat, P. V.; Hue, N. V.; Quang, D. T.; Trung, N. T.; Tung, V. T.; Huynh, L. K.; Nhung, N. T. A., Investigation into SARS-CoV2 Resistance of Compounds in Garlic Essential Oil. ACS Omega 2020.

9. Navneet, B.; Sowmya Ramaswamy, K.; Gopalakrishnan, B.; Arijit, R., De Novo Design of New Chemical Entities (NCES) for SARS-CoV-2 Using Artificial Intelligence. 2020.

10. Goodsell, D. S.; Morris, G. M.; Olson, A. J., Automated docking of flexible ligands: Applications of AutoDock. J. Mol. Recognit. 1996, 9 (1), 1-5.

11. O'Boyle, N. M.; Banck, M.; James, C. A.; Morley, C.; Vandermeersch, T.; Hutchison, G. R., Open Babel: An open chemical toolbox. J Cheminform 2011, 3, 33.

12. Bonomi, M.; Branduardi, D.; Bussi, G.; Camilloni, C.; Provasi, D.; Raiteri, P.; Donadio, D.; Marinelli, F.; Pietrucci, F.; Broglia, R. A.; Parrinello, M., PLUMED: A portable plugin for free-energy calculations with molecular dynamics. Comput. Phys. Commun. 2009, $180(10), 1961-1972$.

13. Spiwok, V. metadynminer: Tools to Read, Analyze and Visualize Metadynamics HILLS Files from 'Plumed', https://CRAN.R-project.org/package=metadynminer, 2019. 
Other files

DNVLive_covid_SI_small.mp4 (46.73 MiB)

view on ChemRxiv - download file 